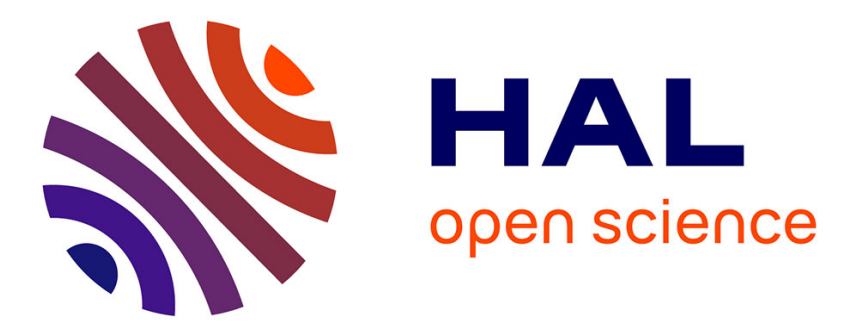

\title{
A second order all Mach number IMEX finite volume solver for the three dimensional Euler equations
}

Walter Boscheri, Giacomo Dimarco, Raphaël Loubère, Maurizio Tavelli, Marie-Hélène Vignal

\section{- To cite this version:}

Walter Boscheri, Giacomo Dimarco, Raphaël Loubère, Maurizio Tavelli, Marie-Hélène Vignal. A second order all Mach number IMEX finite volume solver for the three dimensional Euler equations. Journal of Computational Physics, 2020, 415, pp.109486. 10.1016/j.jcp.2020.109486 . hal-03084457

\section{HAL Id: hal-03084457 \\ https://hal.science/hal-03084457}

Submitted on 22 Dec 2020

HAL is a multi-disciplinary open access archive for the deposit and dissemination of scientific research documents, whether they are published or not. The documents may come from teaching and research institutions in France or abroad, or from public or private research centers.
L'archive ouverte pluridisciplinaire HAL, est destinée au dépôt et à la diffusion de documents scientifiques de niveau recherche, publiés ou non, émanant des établissements d'enseignement et de recherche français ou étrangers, des laboratoires publics ou privés. 


\title{
A second order all Mach number IMEX finite volume solver for the three dimensional Euler equations
}

\author{
Walter Boscheri*a ${ }^{*}$ Giacomo Dimarco $^{\mathrm{a}}$, Raphaël Loubère ${ }^{\mathrm{c}}$, Maurizio Tavelli ${ }^{\mathrm{b}}$, Marie-Hélène Vignal ${ }^{\mathrm{d}}$ \\ ${ }^{a}$ Department of Mathematics and Computer Science, University of Ferrara, Ferrara, Italy \\ ${ }^{b}$ Faculty of Science and Technology, Free University of Bozen, Bolzano, Italy \\ ${ }^{c}$ Univ. Bordeaux, CNRS, Bordeaux INP, Institut de Mathématiques de Bordeaux (IMB), UMR 5251, F-33400, Talence, France \\ ${ }^{d}$ Institut de Mathématiques de Toulouse (IMT); UMR5219, Université de Toulouse ; CNRS, UPS, F-31062 Toulouse Cedex 9, France
}

\begin{abstract}
This article deals with the development of a numerical method for the compressible Euler system valid for all Mach numbers: from extremely low to high regimes. In classical fluid dynamic problems, one faces both situations in which the flow is subsonic, and consequently acoustic waves are very fast compared to the velocity of the fluid, and situations in which the fluid moves at high speed and compressibility may generate shock waves. Standard explicit fluid solvers such as Godunov method fail in the description of both flows due to time step restrictions caused by the stiffness of the equations which leads to prohibitive computational costs. In this work, we develop a new method for the full Euler system of gas dynamics based on partitioning the equations into a fast and a low scale. Such a method employs a time step which is independent of the speed of the pressure waves and works uniformly for all Mach numbers. Cell centered discretization on Cartesian meshes is proposed. Numerical results up to the three dimensional case show the accuracy, the robustness and the effectiveness of the proposed approach.
\end{abstract}

Keywords: All Mach number flow solver, Asymptotic preserving, Implicit-Explicit Runge-Kutta schemes, Incompressible flows, Multidimensional Euler equations.

\section{Introduction}

The equations which model high speed and low speed inviscid flows are well known and have been studied for more than a century [65]. Numerical methods for the solution of fluid dynamics are indeed widely used in industrial and environmental engineering [57, 87]. The design of wind or water turbines, aircraft engines and cars is based on advanced numerical modeling, as well as the study of the evolution of geophysical and environmental flows, like free surface flows in rivers and lakes, oceanic tides or even atmospheric flows. Depending on the physical phenomenon, one can distinguish two different models, namely the compressible Euler equations and the incompressible ones. However, there are situations in which the fluid may be only slightly compressible or situations in which the fluid exhibits very fast pressure waves in some regions of the domain and not in others, or even fast waves are present only for short times compared to the scale of observation. This mainly happens in the context of geophysical and environmental flows, rather than in industrial applications. In all these cases, it is interesting to analyze from a theoretical point of view what happens if one performs the passage from incompressible to compressible scaling and vice versa $[60,61,86,2,66,1]$. It is probably equally interesting from the point of view of the applications to dispose of numerical methods which are able to deal both with the incompressible and the compressible regimes $[52,46,78,96,62,20,44,73,70,47,43,77,71,25,28,26,24,45,16,18,41,59,29,31]$. The compressible Euler equations describe the conservation of density, momentum and energy, while the corresponding incompressible model

\footnotetext{
${ }^{*}$ Corresponding author

Email addresses: walter.boscheri@unife.it (Walter Boscheri*), giacomo.dimarco@unife.it (Giacomo Dimarco), raphael.loubere@math.u-bordeaux.fr (Raphaël Loubère), maurizio.tavelli@unibz . it (Maurizio Tavelli), mhvignal@math. univ-toulouse.fr (Marie-Hélène Vignal)
} 
deals with a constraint on the divergence of the velocity, with an elliptic equation for the pressure and a linear transport equation for the density moving at the average speed of the flow. These two models can be connected by a rescaling procedure in which the Mach number, i.e. the ratio between the flow and the sound speeds, is put into evidence in the compressible model and then is set to zero through a limit analysis. This situation implies a fluid flow almost at rest, and, the pressure waves to move at large speed compared to the average speed of the gas.

Unfortunately, the aforementioned models cannot deal with all occurrences encountered in practical cases. In fact, in many problems, the presence of low and high Mach number flows becomes evident during the study of the phenomena and cannot be predicted before starting the simulation. Thus, one needs to be able to describe these situations with the same numerical scheme since switching the scheme during the time marching is not a strategy that can be followed. However, this causes many difficulties from the numerical point of view because standard numerical methods suffer from the stiffness of the equations, while domain decomposition techniques which may couple the two models are difficult to be set into practice, as pointed out for instance in [7]. Since the problem involves a fast time scale, one possible solution is given by the use of implicit time integrators. On the other hand, it is well known that implicit schemes are not simple to be applied due to the non linearity of the system. Furthermore, the necessity to employ high order in space schemes complicates the realization of such methods. In the recent past, an alternative strategy to treat problems with multiple time scales has been proposed, which consists in treating implicitly only one part of the system to be solved while keeping the remaining explicit. This approach considerably facilitates the development of the schemes since it diminishes the complexity of the non linear systems to be inverted in order to advance the solution in time. We refer to this class of schemes as Implicit-Explicit methods [3, 8, 76], that are a particular case of the so-called partitioned schemes [50]. Thanks to this choice, it is possible to develop numerical schemes which are stable for all values of the fast scale, independently on the prescribed value of the time step. Moreover, if these schemes share also the property of being consistent with the limit model, they are often referred to as Asymptotic Preserving methods. In the context of compressible-incompressible fluids, these techniques have been developed by several authors in the last decade $[27,25,26,24,45,90,16,72,18,31,30]$. A semi-implicit finite volume scheme introduced in [36], which shares similarities with the one here proposed, makes use of a staggered grid spatial discretization. Staggered meshes in the context of semi-implicit schemes for incompressible fluids have also been considered in [91, 93, 11, 12]. In two recent works [31, 30], first and second order Asymptotic Preserving methods have been developed for the low Mach number limit for the simplified isentropic Euler case. In [21] a semiimplicit scheme for atmospheric flows is proposed where the separation of acoustic and advective components of the hyperbolic flux is carried out in the characteristic space, while in [101] a correction to deal with non-hyperbolic flux splitting is presented. High order discontinuous Galerkin methods on staggered grids for compressible flows have been forwarded in [92].

In the present work, we extend the previous study to the full Euler equations with the intent of simulating up to three dimensional flows. Compared to the isentropic Euler situation, here the difficulties are related to search for the minimum number of terms to treat implicitly in the full Euler system, and, to deal with the non linearity of the system which arises in the scheme. All these aspects increase in complexity by the fact that multidimensional problems are solved. Compared to the works $[95,36]$, here we do not use staggered grids, we work in the full three dimensional setting and more important we develop a second order in time method which was not present in the aforementioned references. Moreover, we give evidence, even if formal, of the all Mach properties (Asymptotic Preserving property) of the proposed schemes which was not analysed in [95, 36]. In the following, we first present a first order in time and space method and then we extend our analysis to general second order in time Implicit-Explicit (IMEX) methods coupled with a second order space discretization approach. The time marching technique is based on IMEX Runge Kutta method for the time and TVD second order discretization for the space [64]. In [30], we focused on the analysis of the development of $L^{2}$ and $L^{\infty}$ stable methods and on the differences that arise if one stability condition is assured instead of the other. In this work, we only consider the weaker $L^{2}$ stability requirement for sake of shortness and simplicity.

The article is organized as follows. In section 2, we recall the governing equations, the low Mach scaling and we give the details of the formal passage from the compressible to the incompressible model. In section 3, we introduce a first order scheme which works for all values of the Mach number. We start by discussing the semi discrete in time case in 3.1, then we give the details of the full time and space discretization for the one dimensional case in section 3.2 and we end with the three dimensional first order case in section 3.4. The second order extension in time and space of our method is proposed in section 4, namely the time semi discretization is discussed in section 4.1 while the space 
second order extension in section 4.2. The numerical results showing the effectiveness of the proposed approach are given in section 5. A concluding section finalizes the article.

\section{Governing equations}

We consider a bounded domain $\Omega \in \mathbb{R}^{d}$ with the space dimension $d \in\{1,2,3\}$. The space and time variables are respectively denoted by $x \in \Omega$ and $t \in \mathbb{R}_{+}$. The compressible Euler equations read

$$
\begin{aligned}
& \partial_{t} \rho+\nabla_{\boldsymbol{x}} \cdot \boldsymbol{q}=0, \\
& \partial_{t} \boldsymbol{q}+\nabla_{\boldsymbol{x}} \cdot\left(\frac{\boldsymbol{q} \otimes \boldsymbol{q}}{\rho}\right)+\nabla_{\boldsymbol{x}} p=0, \\
& \partial_{t} E+\nabla_{\boldsymbol{x}} \cdot\left((E+p) \frac{\boldsymbol{q}}{\rho}\right)=0,
\end{aligned}
$$

where $\rho(t, \boldsymbol{x})>0$ is the density of the fluid, $\boldsymbol{q}(t, \boldsymbol{x})=(\rho(t, \boldsymbol{x}) \boldsymbol{u}(t, \boldsymbol{x})) \in \mathbb{R}^{d}$ the momentum, $\boldsymbol{u}(t, \boldsymbol{x})$ represents the velocity vector, $E(t, \boldsymbol{x})$ is the total energy and $p \equiv p(\rho, \boldsymbol{q}, E)$ is the fluid pressure defined by the following equation of state

$$
p \equiv p(\rho, \boldsymbol{q}, E)=(\gamma-1)\left(E-\frac{1}{2} \frac{|\boldsymbol{q}|^{2}}{\rho}\right) .
$$

The parameter $\gamma=c_{p} / c_{v} \geq 1$ is a characterization of the fluid and represents the ratio of specific heats, namely the heat capacity at constant volume $c_{v}$ and at constant pressure $c_{p}$. In the following, the state variables are often gathered into one $(d+2)$-vector $\boldsymbol{Q}=(\rho, \boldsymbol{q}, E)$. The low Mach number limit can be obtained from this model by rescaling the equations in the following way:

$$
\tilde{\rho}=\rho / \rho_{0}, \quad \tilde{\boldsymbol{u}}=\boldsymbol{u} / u_{0}, \quad \tilde{p}=p / p_{0}, \quad \tilde{E}=E / p_{0}, \quad \tilde{\boldsymbol{x}}=\boldsymbol{x} / x_{0}, \quad \tilde{t}=t / t_{0},
$$

where $\rho_{0}, p_{0}, x_{0}, t_{0}$ and $u_{0}=x_{0} / t_{0}$ are typical values encountered in the problems under consideration. Now, by defining $\varepsilon=\rho_{0} u_{0}^{2} / p_{0}$, inserting (5) into system (1)-(4) and omitting the tildes, one obtains the rescaled Euler equations

$$
\begin{aligned}
& \partial_{t} \rho+\nabla_{\boldsymbol{x}} \cdot \boldsymbol{q}=0, \\
& \partial_{t} \boldsymbol{q}+\nabla_{\boldsymbol{x}} \cdot\left(\frac{\boldsymbol{q} \otimes \boldsymbol{q}}{\rho}\right)+\frac{1}{\varepsilon} \nabla_{\boldsymbol{x}} p=0, \\
& \partial_{t} E+\nabla_{\boldsymbol{x}} \cdot\left((E+p) \frac{\boldsymbol{q}}{\rho}\right)=0,
\end{aligned}
$$

and the corresponding rescaled equation of state

$$
p=(\gamma-1)\left(E-\frac{\varepsilon}{2} \frac{|\boldsymbol{q}|^{2}}{\rho}\right)
$$

The quantity $\varepsilon>0$ is related to the Mach number $M$ and the sound speed $c$ through the relation $M=u_{0} / c=\sqrt{\varepsilon / \gamma}$, where $c$ is given by $c^{2}=\left(\frac{\partial p}{\partial \rho}\right)_{s}=\gamma \frac{p}{\rho}, s$ being the entropy [60,68].

The Low-Mach regimes correspond to situations in which the sound speed in the fluid is very high compared to the speed of the fluid itself, and consequently to small values of $\varepsilon$. In such situations, if an explicit scheme is used, the rescaled sound speed dictates a severe CFL stability condition that drastically limits the maximum admissible size of the time step. Indeed, for instance for $d=3$ and setting $\boldsymbol{x}=(x, y, z)$ and $\boldsymbol{u}=(u, v, w)$, the eigenvalues of the Jacobian matrix in the direction $x$ are $\lambda_{1 x}=u-c / \sqrt{\varepsilon}, \lambda_{2 x}=u$ (multiplicity 3) and $\lambda_{3 x}=u+c / \sqrt{\varepsilon}$ (the same results hold for the directions $y$ and $z$ changing $u$ by $v$ and $w$, respectively). The time-step $\Delta t=t^{n+1}-t^{n}$ must then satisfy the CFL condition, which is given by

$$
\Delta t \leq \frac{1}{\frac{\max (|u \pm c / \sqrt{\varepsilon}|)}{\Delta x}+\frac{\max (|v \pm c / \sqrt{\varepsilon}|)}{\Delta y}+\frac{\max (|w \pm c / \sqrt{\varepsilon}|)}{\Delta z}},
$$


where $\Delta x, \Delta y$ and $\Delta z$ are the respective space steps for the different directions. Then, $\Delta t$ is of order $\sqrt{\varepsilon}$ and tends to 0 with $\varepsilon$. Furthermore, even if this constraint is satisfied, it is well known (see [44], [43] or [28]) that explicit schemes suffer from a consistency problem in the limit $\varepsilon \rightarrow 0$. Indeed, they are not capable to capture the right asymptotic regime.

A possible way to bypass these limitations is to use the incompressible Euler equations (IE) obtained as the low Mach number limit of the compressible Euler equations (CE), i.e. $\lim _{\varepsilon \rightarrow 0} C E=I E$. This limit is formally recalled in the next section. However, this solution can be used only if the fluid is incompressible in the entire computational domain. For situations such that compressible zones coexist with incompressible ones, alternative remedies should be studied. This is the case of asymptotic stable schemes which are free of time step constraints related to fast temporal scales. To construct such schemes, it is necessary to determine what are the terms, in the compressible Euler equations, that carry the sound waves information. In [95], it has been shown that the pressure term in the momentum equations and the enthalpy term in the energy equation are those generating these fast sound waves that constrain the time step. Therefore, in the sequel we choose to implicitly discretize these terms to avoid a restrictive stability condition for low Mach number flows.

We end this section by providing notations useful in the sequel. The specific enthalpy $h$ is given by

$$
h=e+p,
$$

where $e$ is the internal energy $e$ given by

$$
e=\frac{p}{\gamma-1}
$$

Then, the total energy is the sum of the internal energy $e$ and the kinetic energy $k=\frac{\varepsilon}{2} \rho|\boldsymbol{u}|^{2}$, that yields the relation

$$
E=e+k=e+\frac{\varepsilon}{2} \rho|\boldsymbol{u}|^{2}
$$

The total energy flux in (3) can be reformulated according to [95] as

$$
\boldsymbol{u}(E+p)=k \boldsymbol{u}+h \boldsymbol{u} .
$$

The compressible Euler equations can be written in expanded form in the three dimensional case by the equations

$$
\boldsymbol{Q}_{t}+\boldsymbol{F}_{x}+\boldsymbol{G}_{y}+\boldsymbol{W}_{z}=\mathbf{0}
$$

where the vectors of conservative variables $\mathbf{Q}$ and the fluxes $(\boldsymbol{F}(\mathbf{Q}), \boldsymbol{G}(\mathbf{Q}), \boldsymbol{W}(\mathbf{Q}))$ explicitly write

$$
\boldsymbol{Q}=\left[\begin{array}{c}
\rho \\
\rho u \\
\rho v \\
\rho w \\
E
\end{array}\right], \quad \boldsymbol{F}(\mathbf{Q})=\left[\begin{array}{c}
\rho u \\
\rho u^{2}+\frac{1}{\varepsilon} p \\
\rho u v \\
\rho u w \\
u(k+h)
\end{array}\right], \quad \boldsymbol{G}(\mathbf{Q})=\left[\begin{array}{c}
\rho v \\
\rho v u \\
\rho v^{2}+\frac{1}{\varepsilon} p \\
\rho v w \\
v(k+h)
\end{array}\right], \quad \boldsymbol{W}(\mathbf{Q})=\left[\begin{array}{c}
\rho w \\
\rho u w \\
\rho v w \\
\rho w^{2}+\frac{1}{\varepsilon} p \\
w(k+h)
\end{array}\right],
$$

with $\boldsymbol{x}=(x, y, z) \in \Omega$ and $\boldsymbol{u}=(u, v, w)$. Before introducing the numerical scheme, we give the details of the formal derivation of the low-Mach (or incompressible) limit of the compressible Euler equations which will be useful in the analysis of the numerical scheme.

\subsection{Low Mach limit and Incompressible Euler equations}

The rigorous low-Mach limit of the compressible Euler system has been widely studied in the last years [2, 60, 61, $66,86]$. In the isentropic case, the solutions are known to converge to the solution of the corresponding incompressible Euler equations independently from the choice of the initial data (well prepared/consistent or not to the limit regime). Results in the case of non-isentropic Euler equations with general initial data can be found in [68] in the free space $\Omega=\mathbb{R}^{d}$, in [1] for an exterior domain and in a bounded toroidal domain in [69]. 
Here, we briefly recall the formal limit for a general bounded domain in the simplified case of well-prepared initial conditions. We supplement system (6)-(9), or equivalently (9)-(12), with the permeability boundary condition

$$
\boldsymbol{u}(t, \boldsymbol{x}) \cdot \boldsymbol{v}(\boldsymbol{x})=0, \forall \boldsymbol{x} \in \partial \Omega, t>0
$$

where $\boldsymbol{v}(\boldsymbol{x})$ denotes the unit outward normal vector to the boundary $\partial \Omega$ of the domain $\Omega$. We consider well prepared initial conditions

$$
(\rho, \boldsymbol{u}, p)(t=0, \boldsymbol{x})=\left(\rho^{0}(\boldsymbol{x}), \boldsymbol{u}^{0}(\boldsymbol{x}), p^{0}(\boldsymbol{x})\right), \forall \boldsymbol{x} \in \Omega,
$$

where

$$
\rho^{0}(\boldsymbol{x}) \geq 0, \quad p^{0}(\boldsymbol{x})=\bar{p}_{0}+\varepsilon \tilde{p}_{0}(\boldsymbol{x}) \geq 0, \quad \boldsymbol{u}^{0}(\boldsymbol{x})=\overline{\boldsymbol{u}}_{0}(\boldsymbol{x})+\sqrt{\varepsilon} \tilde{\boldsymbol{u}}_{0}(\boldsymbol{x}), \quad \nabla_{\boldsymbol{x}} \cdot \overline{\boldsymbol{u}}_{0}(\boldsymbol{x})=0,
$$

where $\bar{p}_{0}$ is a fixed constant. We assume that all quantities $\rho, \boldsymbol{u}, p$ and $E$, solutions of (6)-(9), converge respectively towards some values $\rho_{0}, \boldsymbol{u}_{0}, p_{0}$ and $E_{0}$ when $\varepsilon$ tends to 0 . Then, multiplying the momentum equations and passing to the limit lead to $\nabla_{x} p_{0}=0$ and, consequently, $p_{0}(t, \boldsymbol{x})=p_{0}(t)$. The low Mach limit of the equation of state (9) yields moreover $E_{0}(t, \boldsymbol{x})=E_{0}(t)=p_{0}(t) /(\gamma-1)$. Integrating now the limit of the energy equation (8) on the domain, using the Green formula and the limit permeability condition (16), gives

$$
|\Omega| E_{0}^{\prime}(t)+\left(E_{0}(t)+p_{0}(t)\right) \int_{\partial \Omega} \boldsymbol{u}_{0}(\boldsymbol{x}, t) \cdot \boldsymbol{v}(\boldsymbol{x}) d \sigma(\boldsymbol{x})=|\Omega| E_{0}^{\prime}(t)=0,
$$

where $d \sigma$ is the $d$-1-dimensional Lebesgue measure on $\partial \Omega$. Then, $E_{0}(t)=E_{0}(0)=\bar{p}_{0} /(\gamma-1)$ and $p_{0}(t)=\bar{p}_{0}$.

By considering again the energy equation (8), one notices that the constant limit pressure implies the incompressibility condition $\nabla_{\boldsymbol{x}} \cdot \boldsymbol{u}_{0}=0$. Finally, assuming that $p_{1}=\lim _{\varepsilon \rightarrow 0} \frac{1}{\varepsilon}\left(p-\bar{p}_{0}\right)$ exists, we recover the incompressible limit system [1], that is

$$
\begin{gathered}
\partial_{t} \rho_{0}+\nabla_{\boldsymbol{x}} \cdot \boldsymbol{q}_{0}=0, \\
\partial_{t} \boldsymbol{q}_{0}+\nabla_{\boldsymbol{x}} \cdot\left(\frac{\boldsymbol{q}_{0} \otimes \boldsymbol{q}_{0}}{\rho_{0}}\right)+\nabla_{\boldsymbol{x}} p_{1}=0, \\
\nabla_{\boldsymbol{x}} \cdot \boldsymbol{u}_{0}=0 .
\end{gathered}
$$

We can observe that $p=\bar{p}_{0}+\varepsilon p_{1}+o\left(\varepsilon^{2}\right)$ and that $p_{1}$ is implicitly defined by the constraint $\nabla_{\boldsymbol{x}} \cdot \boldsymbol{u}_{0}=0$ and explicitly given by the equation

$$
-\nabla_{\boldsymbol{x}} \cdot\left(\frac{1}{\rho_{0}} \nabla_{\boldsymbol{x}} p_{1}\right)=\nabla_{\boldsymbol{x}}\left(\left(\boldsymbol{u}_{0} \cdot \nabla_{\boldsymbol{x}}\right) \boldsymbol{u}_{0}\right)
$$

In addition, the density conservation equation reduces to $\partial_{t} \rho_{0}+\boldsymbol{u}_{0} \cdot \nabla_{x} \rho_{0}=0$, which is a pure transport equation at the velocity $\boldsymbol{u}_{0}$. Note that if $\rho_{0}$ is initially constant then $\rho_{0}$ remains constant for all times and the explicit equation for $p_{1}$ becomes

$$
-\Delta_{x} p_{1}=\nabla_{\boldsymbol{x}}:\left(\rho_{0} \boldsymbol{u}_{0} \otimes \boldsymbol{u}_{0}\right) .
$$

The incompressible system can also be expressed using the energy variable, remarking that

$$
\lim _{\varepsilon \rightarrow 0} \frac{1}{\varepsilon}\left(E-\frac{\bar{p}_{0}}{\gamma-1}\right)=\lim _{\varepsilon \rightarrow 0} \frac{1}{\varepsilon}\left(E-\bar{E}_{0}\right)=\frac{1}{\gamma-1} \lim _{\varepsilon \rightarrow 0}\left(\frac{1}{\varepsilon}\left(p-\bar{p}_{0}\right)+\frac{1}{2} \frac{|\boldsymbol{q}|^{2}}{\rho}\right)=\frac{p_{1}}{\gamma-1}+\frac{1}{2} \frac{\left|\boldsymbol{q}_{0}\right|^{2}}{\rho_{0}} .
$$

Then, defining $E_{1}$ by $E_{1}=\frac{p_{1}}{\gamma-1}+\frac{1}{2} \frac{\left|\boldsymbol{q}_{0}\right|^{2}}{\rho_{0}}$, we obtain $E=\bar{E}_{0}+\varepsilon E_{1}+o\left(\varepsilon^{2}\right)$ and system (19)-(21) becomes

$$
\begin{aligned}
& \partial_{t} \rho_{0}+\nabla_{\boldsymbol{x}} \cdot \boldsymbol{q}_{0}=0 \\
& \partial_{t} \boldsymbol{q}_{0}+\nabla_{\boldsymbol{x}} \cdot\left(\frac{\boldsymbol{q}_{0} \otimes \boldsymbol{q}_{0}}{\rho_{0}}\right)+(\gamma-1) \nabla_{\boldsymbol{x}} \tilde{E}_{1}-\frac{\gamma-1}{2} \nabla_{\boldsymbol{x}}\left(\frac{\left|\boldsymbol{q}_{0}\right|^{2}}{\rho_{0}}\right)=0, \\
& \quad \nabla_{\boldsymbol{x}} \cdot \boldsymbol{u}_{0}=0
\end{aligned}
$$




\section{A first order numerical scheme}

In this section, we introduce and discuss a first order in time and space discretization of the Euler equations (14). We start from a semi discretization in time in the general $d$-dimensional space, then we derive a full spacetime discretization in one space dimension and we end by giving the details of the full discretized three dimensional algorithm.

\subsection{A first order time discretization}

In this part, we discuss the semi-implicit time discretization we decide to follow and we study its properties. The class of schemes considered hereafter belong to the so-called IMEX (IMplicit-EXplicit) time discretization methods (see [25, 26, 90, 31, 30, 8, 10, 9, 3, 76]). In particular, following [95], the fluxes in the governing equations (15) are split into a non stiff part $\left(\boldsymbol{F}^{e x}, \boldsymbol{G}^{e x}, \boldsymbol{W}^{e x}\right)$, which will be discretized explicitly, and a stiff part $\left(\boldsymbol{F}^{i m}, \boldsymbol{G}^{i m}, \boldsymbol{W}^{i m}\right)$, which will be discretized implicitly. This partition write

$$
\begin{aligned}
\boldsymbol{F} & =\boldsymbol{F}^{e x}+\boldsymbol{F}^{i m}, \quad \boldsymbol{G}=\boldsymbol{G}^{e x}+\boldsymbol{G}^{i m}, \quad \boldsymbol{W}=\boldsymbol{W}^{e x}+\boldsymbol{W}^{i m}, \\
& =\left[\begin{array}{c}
\rho u \\
\rho u^{2} \\
\rho u v \\
\rho u w \\
u k
\end{array}\right]+\left[\begin{array}{c}
0 v \\
\frac{p}{\varepsilon} \\
0 \\
0 \\
h u
\end{array}\right], \quad=\left[\begin{array}{c}
0 \\
\rho v u \\
\rho v^{2} \\
\rho v w \\
v k
\end{array}\right]+\left[\begin{array}{c}
\rho w \\
p \\
\varepsilon \\
0 \\
h v u \\
\rho w v \\
\rho w^{2} \\
w k
\end{array}\right], \quad\left[\begin{array}{c}
0 \\
0 \\
0 \\
\frac{p}{\varepsilon} \\
h w
\end{array}\right] .
\end{aligned}
$$

The explicit fluxes contribute to the advection sub-system, while the implicit ones correspond to the pressure (or sound) waves in the fluid. In [95] a detailed study of the eigenstructure of both explicit-advection and implicit-pressure sub-systems prove that both are hyperbolic systems. Therefore, the problem can be recast as

$$
\boldsymbol{Q}_{t}+\left(\boldsymbol{F}^{e x}+\boldsymbol{F}^{i m}\right)_{x}+\left(\boldsymbol{G}^{e x}+\boldsymbol{G}^{i m}\right)_{y}+\left(\boldsymbol{W}^{e x}+\boldsymbol{W}^{i m}\right)_{z}=\mathbf{0},
$$

that yields the first order in time semi-discretization

$$
\underbrace{\boldsymbol{Q}^{n+1}+\Delta t\left(\boldsymbol{F}_{x}^{i m}\left(\mathbf{Q}^{n+1}\right)+\boldsymbol{G}_{y}^{i m}\left(\mathbf{Q}^{n+1}\right)+\boldsymbol{W}_{z}^{i m}\left(\mathbf{Q}^{n+1}\right)\right)}_{\text {Implicit }}=\underbrace{\mathbf{Q}^{n}-\Delta t\left(\boldsymbol{F}_{x}^{e x}\left(\mathbf{Q}^{n}\right)+\boldsymbol{G}_{y}^{e x}\left(\mathbf{Q}^{n}\right)+\boldsymbol{W}_{z}^{e x}\left(\mathbf{Q}^{n}\right)\right)}_{\text {Explicit }} .
$$

For the sake of clarity and compact notation in the description of the methods, let us call $\mathcal{L}_{i}=\left(\boldsymbol{F}^{i}, \boldsymbol{G}^{i}, \boldsymbol{W}^{i}\right)$ the implicit operator and $\mathcal{L}_{e}=\left(\boldsymbol{F}^{e}, \boldsymbol{G}^{e}, \boldsymbol{W}^{e}\right)$ the explicit one. In this way, the scheme (27) is rewritten as

$$
\boldsymbol{Q}^{n+1}=\boldsymbol{Q}^{n}-\Delta t \nabla_{\boldsymbol{x}} \cdot \mathcal{L}_{i}\left(\boldsymbol{Q}^{n+1}\right)-\Delta t \nabla_{\boldsymbol{x}} \cdot \mathcal{L}_{e}\left(\boldsymbol{Q}^{n}\right) .
$$

We show in Lemma 1 that this choice is enough to ensure the asymptotically consistency of the semi-discrete scheme, i.e. the consistency with the incompressible limit. In $[31,30]$ it is has been shown on a simplified problem mimicking the isentropic model that stability can be also ensured with a CFL related to the eigenvalues of the explicit part of the matrix. We believe that such a study can be extended to the present system in its linearised form. Nevertheless, such stability analysis is not simple and it is subject of ongoing investigation. Instead, here, we show in the numerical results presented in section 5 that stability is indeed assured independently on the speed of the fast pressure waves through numerical evidences.

The example described in the previous section shows how the compressible equations reduce to the incompressible ones in the asymptotic limit. The construction of numerical schemes which are capable to capture a limit model starting from another set of equations is closely connected with the notion of asymptotic-preserving schemes. Figure 1 , originally proposed in [56], provides a a visual explanation of this concept. Here, in agreement with [26, 27, 76], we give the following definitions of asymptotic preserving methods for system (15).

Definition 1. A consistent time discretization method for (15) supplemented by the equation of state (9) of stepsize $\Delta t$ is said 


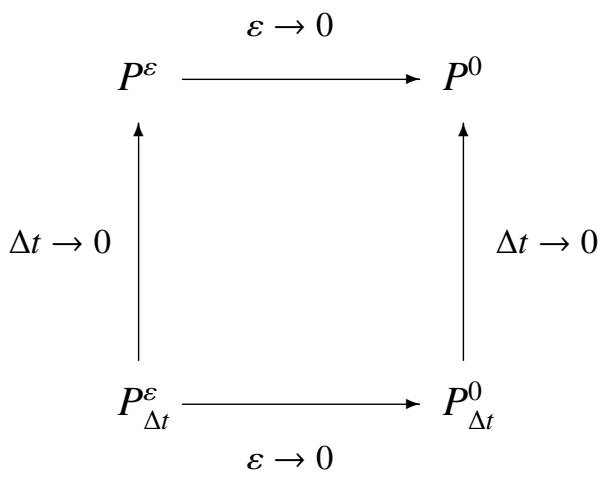

Figure 1: $P^{\varepsilon}$ is the original singular perturbation problem, i.e. the compressible Euler model, and $P_{\Delta t}^{\varepsilon}$ its numerical approximation characterized by a discretization parameter $\Delta t$. The asymptotic-preserving (AP) property corresponds to the request that for a given $\Delta t$ independent of $\varepsilon$, when $\varepsilon \rightarrow 0, P_{\Delta t}^{\varepsilon}$ is a consistent and stable discretization of $P^{0}$, which is the incompressible Euler model.

- Strong asymptotic preserving if, independently of the initial data, it is asymptotically stable and consistent. That is if, it is stable for time steps $\Delta t$ which can be chosen independently of $\varepsilon$, and it becomes a consistent time discretization method for the limit system (19)-(21) in the limit $\varepsilon \rightarrow 0$.

- Weak asymptotic preserving if, it is asymptotically stable and it is asymptotically consistent when the initial data are well prepared to the limit equations. That is if, it is stable for time steps $\Delta t$ which can be chosen independently of $\varepsilon$, and if it becomes a consistent time discretization method for the limit system (19)-(21) in the limit $\varepsilon \rightarrow 0$.

These definitions do not imply that the scheme preserves the order of accuracy in time in the stiff limit $\varepsilon \rightarrow 0$. In this latter case we say that the scheme is asymptotically accurate (AA). The definition of consistent or well-prepared initial data is given in the following.

Definition 2. The initial data for equation (15) and the relative equation of state (9) are said consistent or wellprepared [86] if

$$
p_{\varepsilon}(t=0, \boldsymbol{x})=\bar{p}_{0}+\varepsilon p_{1}(\boldsymbol{x}), \quad \bar{p}_{0}, p_{1}(\boldsymbol{x}) \in \mathbb{R}^{+}, \quad \boldsymbol{u}_{\varepsilon}(t=0, \boldsymbol{x})=\boldsymbol{u}_{0}(\boldsymbol{x})+\sqrt{\varepsilon} \boldsymbol{u}_{1}(\boldsymbol{x}), \quad \nabla \cdot \boldsymbol{u}_{0}(\boldsymbol{x})=0 .
$$

That is, if the pressure field reduces to a constant value in the limit, and the divergence of the velocity field is equal to zero in the same limit.

In what follows, we will consider the development of asymptotic preserving and asymptotically accurate schemes using the general setting of IMEX Runge-Kutta methods [3, 75]. We start by giving a formal proof that this first order in time explicit-implicit approach (28) provides a consistent discretization of the limit model when $\varepsilon \rightarrow 0$.

Lemma 1. We denote by $\left(\rho_{\varepsilon}^{n}(\boldsymbol{x}), \boldsymbol{u}_{\varepsilon}^{n}(\boldsymbol{x}), E_{\varepsilon}^{n}(\boldsymbol{x})\right)_{n \geq 1}$ the solution of the semi discrete numerical scheme (28) with the permeability boundary condition $\boldsymbol{u}^{n}(\boldsymbol{x}) \cdot \boldsymbol{v}(\boldsymbol{x})=0, \forall \boldsymbol{x} \in \partial \Omega, \forall n \geq 0$, Then:

1. assuming that the discrete quantities $\left(\rho_{\varepsilon}^{n}(\boldsymbol{x}), \boldsymbol{u}_{\varepsilon}^{n}(\boldsymbol{x}), E_{\varepsilon}^{n}(\boldsymbol{x})\right)_{n \geq 1}$ converges towards some finite values when $\varepsilon \rightarrow 0$ and assuming that initial data are well prepared to the incompressible regime, i.e. for all $\boldsymbol{x} \in \Omega, p^{0}(\boldsymbol{x})=\bar{p}_{0} \in$ $\mathbb{R}^{+}$and $\nabla \cdot \boldsymbol{u}^{0}(\boldsymbol{x})=\nabla \cdot \boldsymbol{u}_{0}(\boldsymbol{x})=0$, the semi discrete numerical scheme (28) is asymptotically consistent, i.e. in the limit $\varepsilon \rightarrow 0$ it becomes a consistent discretization of system (19)-(21). This discretization is given for all 
$n \geq 0$ by

$$
\begin{aligned}
& \frac{\rho^{n+1}(\boldsymbol{x})-\rho^{n}(\boldsymbol{x})}{\Delta t}+\boldsymbol{u}^{n}(\boldsymbol{x}) \cdot \nabla_{x} \rho^{n}(\boldsymbol{x})=0, \\
& \frac{\left(\rho^{n+1} \boldsymbol{u}^{n+1}\right)(\boldsymbol{x})-\left(\rho^{n} \boldsymbol{u}^{n}\right)(\boldsymbol{x})}{\Delta t}+\nabla_{\boldsymbol{x}} \cdot\left(\left(\rho^{n} \boldsymbol{u}^{n}\right)(\boldsymbol{x}) \otimes \boldsymbol{u}^{n}(\boldsymbol{x})\right)+\nabla_{x} p_{1}^{n+1}(\boldsymbol{x})=0, \\
& \nabla_{\boldsymbol{x}} \cdot \boldsymbol{u}^{n+1}(\boldsymbol{x})=0 .
\end{aligned}
$$

2. For general initial data, the semi discrete numerical scheme (28) is strongly asymptotically consistent from the second time iteration $(n \geq 2)$. This means that in the limit $\varepsilon \rightarrow 0$ it becomes a consistent discretization of system (19)-(21). This is given for all $n \geq 1$ by equation (30) and

$$
\begin{aligned}
& \frac{\rho^{n+1}(\boldsymbol{x})-\rho^{n}(\boldsymbol{x})}{\Delta t}+\nabla_{x} \cdot\left(\left(\rho^{n} \boldsymbol{u}^{n}\right)(\boldsymbol{x})\right)=0, \quad \text { if } n=1 \\
& \frac{\rho^{n+1}(\boldsymbol{x})-\rho^{n}(\boldsymbol{x})}{\Delta t}+\boldsymbol{u}^{n} \cdot \nabla_{x}\left(\rho^{n}(\boldsymbol{x})\right)=0, \quad \text { if } n \geq 2 \\
& \nabla_{\boldsymbol{x}} \cdot \boldsymbol{u}^{n+1}(\boldsymbol{x})= \begin{cases}0, & \text { if } n \geq 2, \\
-\frac{\int_{\Omega} \bar{p}^{0}(\boldsymbol{x}) d \boldsymbol{x}-|\Omega| \bar{p}^{0}(\boldsymbol{x})}{\Delta t \gamma \int_{\Omega} \bar{p}^{0}(\boldsymbol{x}) d \boldsymbol{x}}, & \text { if } n=1 .\end{cases}
\end{aligned}
$$

Proof. We assume that for all $n \geq 1$ all quantities $\rho_{\varepsilon}^{n}(\boldsymbol{x}), \boldsymbol{u}_{\varepsilon}^{n}(\boldsymbol{x}), p_{\varepsilon}^{n}(\boldsymbol{x})$ and $E_{\varepsilon}^{n}(\boldsymbol{x})$, which are solutions of (28), converge respectively towards some values $\rho^{n}(\boldsymbol{x}), \boldsymbol{u}^{n}(\boldsymbol{x}), p^{n}(\boldsymbol{x})$ and $E^{n}(\boldsymbol{x})$ when $\varepsilon$ tends to 0 . Under this hypothesis, multiplying by $\varepsilon$ the momentum equations of (28) and letting $\varepsilon$ tend to zero, gives, for all $n \geq 0, \nabla_{x} p^{n+1}(\boldsymbol{x})=0$, i.e. the pressure at time index $(n+1)$ is constant independently on the initial data. From equation (28), we have

$$
E_{\varepsilon}^{n+1}(\boldsymbol{x})=e_{\varepsilon}^{n+1}(\boldsymbol{x})+\frac{\varepsilon}{2} \rho_{\varepsilon}^{n+1}(\boldsymbol{x})\left|\boldsymbol{u}_{\varepsilon}^{n+1}(\boldsymbol{x})\right|^{2}=\frac{p_{\varepsilon}^{n+1}(\boldsymbol{x})}{(\gamma-1)}+\frac{\varepsilon}{2} \rho_{\varepsilon}^{n+1}(\boldsymbol{x})\left|\boldsymbol{u}_{\varepsilon}^{n+1}(\boldsymbol{x})\right|^{2}
$$

that gives $E^{n+1}(\boldsymbol{x})=p^{n+1}(\boldsymbol{x}) /(\gamma-1)$ for all $n \geq 1$, in the limit $\varepsilon \rightarrow 0$. The limit of the energy equation yields, for all $n \geq 1$, all $\boldsymbol{x} \in \Omega$ and all initial data

$$
E^{n+1}(\boldsymbol{x})=E^{n}(\boldsymbol{x})-\Delta t\left(e^{n+1}(\boldsymbol{x})+p^{n+1}(\boldsymbol{x})\right) \nabla_{\boldsymbol{x}} \cdot \boldsymbol{u}^{n+1}(\boldsymbol{x}) .
$$

Thus, integrating this equation on the domain, using the Green formula and the limit of the permeability boundary condition leads to

$$
|\Omega| E^{n+1}(\boldsymbol{x})=\int_{\Omega} E^{n}(\boldsymbol{x}) d \boldsymbol{x}-\Delta t\left(e^{n+1}(\boldsymbol{x})+p^{n+1}(\boldsymbol{x})\right) \int_{\partial \Omega} \boldsymbol{u}^{n+1}(\boldsymbol{x}) \cdot \boldsymbol{v}(\boldsymbol{x}) d \sigma(\boldsymbol{x})=\int_{\Omega} E^{n}(\boldsymbol{x}) d \boldsymbol{x} .
$$

Now, if initial data are well prepared, this implies that for the first time step we have

$$
\frac{p^{1}(\boldsymbol{x})}{\gamma-1}=E^{1}(\boldsymbol{x})=\frac{1}{|\Omega|} \int_{\Omega} E^{0}(\boldsymbol{x}) d \boldsymbol{x}=\frac{1}{|\Omega|} \int_{\Omega} \frac{p^{0}(\boldsymbol{x})}{\gamma-1} d \boldsymbol{x}=\frac{1}{|\Omega|} \int_{\Omega} \frac{\bar{p}_{0}}{\gamma-1} d \boldsymbol{x}=\frac{\bar{p}_{0}}{\gamma-1} .
$$

Consequently, for all $n \geq 1$, both pressure and energy remain constant: $p^{n+1}=\bar{p}_{0}, E^{n+1}=\bar{p}_{0} /(\gamma-1)$. Using again (35), gives now for all $n \geq 0, \nabla_{x} \cdot \boldsymbol{u}^{n+1}(\boldsymbol{x})=0$, i.e. equations (29)-(31). Assuming finally that the following limit for $\varepsilon \rightarrow 0$ exists

$$
\lim _{\varepsilon \rightarrow 0} \frac{1}{\varepsilon}\left(p_{\varepsilon}^{n+1}-p^{n+1}\right)=\lim _{\varepsilon \rightarrow 0} \frac{1}{\varepsilon}\left(p \varepsilon,{ }^{n+1}-\frac{1}{|\Omega|} \int_{\Omega} \bar{p}^{0}(\boldsymbol{x}) d \boldsymbol{x}\right)=p_{1}^{n+1}
$$

and passing to the limit into the momentum equation of (28), we obtain (30).

We discuss now the case in which initial data are not well prepared to the limit incompressible system. In this case, we have again that for $n=1$ the energy is projected over a constant state: $=E^{1}(\boldsymbol{x})=\frac{1}{|\Omega|} \int_{\Omega} E^{0}(\boldsymbol{x}) d \boldsymbol{x}$. However, 
since the initial condition does not force constant values for pressure and consequently for the energy, we have that the incompressibility condition is not preserved neither at the beginning $\nabla_{\boldsymbol{x}} \cdot \boldsymbol{u}^{0} \neq 0$ nor for the first time step. This is given precisely by

$$
\nabla_{\boldsymbol{x}} \cdot \boldsymbol{u}^{1}=-\frac{E^{1}-E^{0}(\boldsymbol{x})}{\Delta t\left(e^{1}+p^{1}\right)} .
$$

Nevertheless, from $n=2$, following equation (36), we have $E^{n+1}=E^{n}$ which means that energy is constant in space and thus $\nabla_{\boldsymbol{x}} \cdot \boldsymbol{u}^{n+1}(\boldsymbol{x})=0$, i.e. we are solving equations (29)-(31). The equation for $p_{1}^{n+1}$ is obtained as done for the case of well prepared initial data passing to the limit in equation (28). This concludes the proof.

In the next section, we give the details of the one dimensional time and space discretization and we discuss in practice how we solve the implicit terms in the non linear system (28).

\subsection{A full time and space Asymptotic Preserving scheme for the Euler equations}

We present now the full space and time discretization of system (26) for the one-dimensional case. We will then extend this scheme in section 3.4 to the full three dimensional setting. In one space dimension, the governing equations read

$$
\mathbf{Q}_{t}+\boldsymbol{F}_{x}=\mathbf{0}, \quad x \in \Omega \subset \mathbb{R}, \quad t \in \mathbb{R}_{0}^{+}, \quad \mathbf{Q} \in \mathbb{R}^{3},
$$

with the vector of conservative variables given by $\mathbf{Q}=[\rho, \rho u, E]^{T}$. The flux vector is split into an explicit and implicit part according to (25), hence obtaining

$$
\boldsymbol{F}=\boldsymbol{F}^{e x}+\boldsymbol{F}^{i m}=\left[\begin{array}{c}
\rho u \\
\rho u^{2} \\
u k
\end{array}\right]+\left[\begin{array}{c}
0 \\
\frac{p}{\varepsilon} \\
\frac{h}{\rho} q
\end{array}\right] .
$$

The computational domain reduces to $\Omega=\left[x_{\min }, x_{\max }\right]$ and it is discretized with a total number $N_{x}$ of control volumes of uniform size $\Delta x=\frac{x_{\max }-x_{\min }}{N_{x}}$. A cell-centered discretization is used, where index $i \in\left[1, N_{x}\right]$ denotes the cell, while $i \pm 1 / 2$ refers to the interface between cell $i$ and $i \pm 1$, respectively. The cell center is located at $x_{i}$ and the interface is consistently located at $x_{i+1 / 2}=\frac{1}{2}\left(x_{i}+x_{i+1}\right)=x_{i}+\Delta x / 2$. Because of the Implicit-Explicit approach, we choose a time step which is restricted by a CFL condition that is only driven by the eigenvalues of explicit part and, as such, it is only dependent on the fluid velocity $u$, and not by the rescaled sound speed $\frac{c}{\sqrt{\varepsilon}}$, that is

$$
\Delta t \leq \mathrm{CFL} \frac{\Delta x}{\max _{i}\left|2 u_{i}^{n}\right|},
$$

thus making our numerical method particularly well suited for the discretization of low Mach number flows. We stress that a similar approach but on staggered grids has been recently proposed in [36] for general equations of state.

Let us introduce the explicit operator $F^{e}\left[m_{i}^{n}\right]$ which applies to a generic cell quantity $m_{i}^{n}$ :

$$
F^{e}\left[m_{i}^{n}\right]=m_{i}^{n}-\frac{\Delta t}{\Delta x}\left(f_{i+1 / 2}^{m}-f_{i-1 / 2}^{m}\right),
$$

where the numerical fluxes $f_{i \pm 1 / 2}^{m}$ are chosen to be of Rusanov-type

$$
\begin{array}{ll}
f_{i+1 / 2}^{m}=\frac{1}{2}\left(f\left(m_{i+1}^{n}\right)+f\left(m_{i}^{n}\right)\right)-\frac{1}{2}\left|a_{i+1 / 2}^{n}\right|\left(m_{i+1}^{n}-m_{i}^{n}\right), & a_{i+1 / 2}^{n}=\max \left(\left|f^{\prime}\left(m_{i+1}^{n}\right)\right|,\left|f^{\prime}\left(m_{i}^{n}\right)\right|\right), \\
f_{i-1 / 2}^{m}=\frac{1}{2}\left(f\left(m_{i}^{n}\right)+f\left(m_{i-1}^{n}\right)\right)-\frac{1}{2}\left|a_{i-1 / 2}^{n}\right|\left(m_{i}^{n}-m_{i-1}^{n}\right), & a_{i-1 / 2}^{n}=\max \left(\left|f^{\prime}\left(m_{i}^{n}\right)\right|,\left|f^{\prime}\left(m_{i-1}^{n}\right)\right|\right),
\end{array}
$$


with $f(\cdot)$ being the physical flux and $\left|f^{\prime}\left(m_{i}^{n}\right)\right|$ denoting the maximum eigenvalue of the Jacobian matrix associated to the flux $f(\cdot)$, i.e. the maximum speed of the flow $u$ at the interface at time index $n$, since we have separated the pressure and the average speed contributions. The governing system (38) with the flux splitting (39) is then discretized using (41) for $\boldsymbol{F}^{e x}$ and a centred numerical flux for the implicit part $\boldsymbol{F}^{i m}$. Following [31], we believe that this choice is sufficient for a obtaining an $L^{2}$ stable scheme. We postpone such a study to future investigations.

In details, the resulting numerical scheme reads as follows.

1. Density equation with $m_{i}^{n} \equiv \rho_{i}^{n}$ :

$$
\rho_{i}^{n+1}=F^{e}\left[\rho_{i}^{n}\right]
$$

2. Momentum equation with $m_{i}^{n} \equiv q_{i}^{n}=(\rho u)_{i}^{n}$ :

$$
q_{i}^{n+1}=F^{e}\left[q_{i}^{n}\right]-\frac{\Delta t}{2 \varepsilon \Delta x}\left(p_{i+1}^{n+1}-p_{i-1}^{n+1}\right),
$$

where the implicit discretization of the pressure corresponds to the use of the Rusanov flux without numerical dissipation.

3. Energy equation with $m_{i}^{n} \equiv k_{i}^{n}$ :

$$
E_{i}^{n+1}=F^{e}\left[E_{i}^{n}\right]-\frac{\Delta t}{2 \Delta x}\left(\tilde{h}_{i+1 / 2}^{n+1}\left(q_{i+1}^{n+1}+q_{i}^{n+1}\right)-\tilde{h}_{i-1 / 2}^{n+1}\left(q_{i}^{n+1}+q_{i-1}^{n+1}\right)\right),
$$

where the enthalpy on the interfaces is defined as

$$
\tilde{h}_{i+1 / 2}^{n+1}=\frac{\frac{h_{i}^{n+1}}{\rho_{i}^{n+1}} q_{i}^{n+1}+\frac{h_{i+1}^{n+1}}{\rho_{i+1}^{n+1}} q_{i+1}^{n+1}}{q_{i}^{n+1}+q_{i+1}^{n+1}} .
$$

If the fluid is at rest, thus the denominator vanishes in (46), the enthalpy at the interface is simply computed by the arithmetic average: $\tilde{h}_{i+1 / 2}^{n+1}=\frac{1}{2}\left(\frac{h_{i}^{n+1}}{\rho_{i}^{n+1}}+\frac{h_{i+1}^{n+1}}{\rho_{i+1}^{n+1}}\right)$. Note that the implicit flux in the discrete energy equation (45) is not a standard centred approximation but instead it corresponds to a mean centred approximation. This choice is enough to avoid a checkerboard effect in the resulting elliptic equation for the pressure [84] we will derive later on in this article.

The proposed numerical method is built along the lines of [36] and it leads to a non linear system to be solved in order to advance the solution in time from $n$ to $(n+1)$. This solution can be obtained by splitting the total energy $E_{i}^{n+1}$ into an internal and a kinetic contribution according to (12). Then, one can insert the momentum equation (44) into the energy equation (45) obtaining

$$
\begin{aligned}
e_{i}^{n+1}+k_{i}^{n+1} & =F^{e}\left[E_{i}^{n}\right] \\
& -\frac{1}{2} \frac{\Delta t}{\Delta x} \tilde{h}_{i+1 / 2}^{n+1}\left[\left(F^{e}\left[q_{i+1}^{n}\right]+F^{e}\left[q_{i}^{n}\right]\right)-\frac{1}{2} \frac{\Delta t}{\varepsilon \Delta x}\left(p_{i+2}^{n+1}-p_{i}^{n+1}+p_{i+1}^{n+1}-p_{i-1}^{n+1}\right)\right] \\
& +\frac{1}{2} \frac{\Delta t}{\Delta x} \tilde{h}_{i-1 / 2}^{n+1}\left[\left(F^{e}\left[q_{i}^{n}\right]+F^{e}\left[q_{i-1}^{n}\right]\right)-\frac{1}{2} \frac{\Delta t}{\varepsilon \Delta x}\left(p_{i+1}^{n+1}-p_{i-1}^{n+1}+p_{i}^{n+1}-p_{i-2}^{n+1}\right)\right],
\end{aligned}
$$

where the kinetic energy at time $t^{n}$ is computed as

$$
k_{i}^{n+1}=\frac{\varepsilon}{2} \rho_{i}^{n+1}\left(\frac{q_{i}^{n+1}}{\rho_{i}^{n+1}}\right)^{2} .
$$

In the energy equation (47), the variables which are already known at time $t^{n+1}$ are the density $\rho_{i}^{n+1}$, because the continuity equation is solved explicitly by (43), and the explicit operators $F^{e}\left[q_{i}^{n}\right]$ and $F^{e}\left[E_{i}^{n}\right]$. The resulting system of equations is strongly nonlinear. In order to reduce this non linearity, one can first observe that for an ideal gas, 
the internal energy is a linear function of $p$, namely $e=\frac{p}{\gamma-1}$, according to (11). Then, one can rely on a Picard algorithm to converge to the solution at time $t^{n+1}$ by selecting only the pressure terms as implicit ones in the iterative method. Thanks to this strategy, the following mildly nonlinear system is obtained for the pressure $p_{i}^{n+1, r+1}$ as the only unknown at each Picard iteration $r$ :

$$
\begin{aligned}
& -\frac{1}{4} \frac{\Delta t^{2}}{\Delta x} \tilde{h}_{i+1 / 2}^{n+1, r} p_{i+2}^{n+1, r+1}-\frac{1}{4} \frac{\Delta t^{2}}{\Delta x}\left(\tilde{h}_{i+1 / 2}^{n+1, r}-\tilde{h}_{i-1 / 2}^{n+1, r}\right) p_{i+1}^{n+1, r+1} \\
& +\left(\frac{\varepsilon \Delta x}{\gamma-1}+\frac{1}{4} \frac{\Delta t^{2}}{\Delta x}\left(\tilde{h}_{i+1 / 2}^{n+1, r}+\tilde{h}_{i-1 / 2}^{n+1, r}\right)\right) p_{i}^{n+1, r+1} \\
& +\frac{1}{4} \frac{\Delta t^{2}}{\Delta x}\left(\tilde{h}_{i+1 / 2}^{n+1, r}-\tilde{h}_{i-1 / 2}^{n+1, r}\right) p_{i-1}^{n+1, r+1}-\frac{1}{4} \frac{\Delta t^{2}}{\Delta x} \tilde{h}_{i-1 / 2}^{n+1, r} p_{i-2}^{n+1, r+1}=\varepsilon \Delta x b_{i}^{r},
\end{aligned}
$$

where we have multiplied by $\varepsilon$ each sides of the equations, and $b_{i}^{r}$ is the known right hand side:

$$
b_{i}^{r}=\left(F^{e}\left[E_{i}^{n}\right]-k_{i}^{n+1, r}\right)-\frac{\Delta t}{2 \Delta x}\left(\tilde{h}_{i+1 / 2}^{n+1, r} F^{e}\left[q_{i+1}^{n}\right]+\left(\tilde{h}_{i+1 / 2}^{n+1, r}-\tilde{h}_{i-1 / 2}^{n+1, r}\right) F^{e}\left[q_{i}^{n}\right]-\tilde{h}_{i-1 / 2}^{n+1, r} F^{e}\left[q_{i-1}^{n}\right]\right) .
$$

The scope of the next Lemma is to prove that the system (49) admits a unique solution for all $\varepsilon>0$.

Lemma 2. For simplicity, in the following proof, we omit all the exponents in the variables. Let $b_{i}$ for all $i=$ $1, \cdots, N_{x}$, we consider the solution for the pressure field $\left(p_{i}\right)_{-1 \leq i \leq N_{x}+2}$ of system (49). We assume the following boundary conditions: $p_{0}=p_{1}, p_{-1}=p_{2}, p_{N_{x}+1}=p_{N_{x}}$ and $p_{N_{x}+2}=p_{N_{x}-1}$. For this choice, we have

$$
\frac{\Delta x \varepsilon}{2(\gamma-1)} \sum_{i=1}^{N_{x}}\left(\frac{p_{i}+p_{i+1}}{2}\right)^{2}+\left(\frac{p_{i}+p_{i-1}}{2}\right)^{2}+\frac{\Delta t^{2}}{2 \Delta x} \sum_{i=1}^{N_{x}-1} \tilde{h}_{i+1 / 2}\left(\frac{p_{i+2}+p_{i+1}}{2}-\frac{p_{i}+p_{i-1}}{2}\right)^{2} \leq \Delta x \varepsilon(\gamma-1) \sum_{i=1}^{N_{x}}\left|b_{i}\right|^{2},
$$

and if $\tilde{h}_{i+1 / 2} \geq 0$ for all $i=1, \cdots, N_{x}-1$, the system is well-posed, i.e. $b_{i}=0, \forall i=1, \cdots, N_{x}$ implies $p_{i}=0, \forall i=$ $0, \cdots, N_{x}+2$.

Proof. System (49) can be rewritten for all $i=1, \cdots, N_{x}$ as

$$
\frac{\varepsilon \Delta x}{\gamma-1} p_{i}-\frac{\Delta t^{2}}{2 \Delta x}\left[\tilde{h}_{i+1 / 2}\left(\frac{p_{i+2}+p_{i+1}}{2}-\frac{p_{i}+p_{i-1}}{2}\right)-\tilde{h}_{i-1 / 2}\left(\frac{p_{i+1}+p_{i}}{2}-\frac{p_{i-1}+p_{i-2}}{2}\right)\right]=\varepsilon \Delta x b_{i} .
$$

Multiplying this equation by $\left(p_{i}+p_{i-1}\right) / 2+\left(p_{i+1}+p_{i}\right) / 2$ and summing over the index $i$, gives

$$
\begin{aligned}
\frac{\varepsilon}{\gamma-1} \sum_{i=1}^{N_{x}} \Delta x p_{i} & \left(\frac{p_{i}+p_{i-1}}{2}+\frac{p_{i+1}+p_{i}}{2}\right)-\frac{\Delta t^{2}}{2 \Delta x}\left[\sum_{i=1}^{N_{x}} \tilde{h}_{i+1 / 2}\left(\frac{p_{i+2}+p_{i+1}}{2}-\frac{p_{i}+p_{i-1}}{2}\right)\left(\frac{p_{i}+p_{i-1}}{2}+\frac{p_{i+1}+p_{i}}{2}\right)\right. \\
& \left.-\sum_{i=1}^{N_{x}} \tilde{h}_{i-1 / 2}\left(\frac{p_{i+1}+p_{i}}{2}-\frac{p_{i-1}+p_{i-2}}{2}\right)\left(\frac{p_{i}+p_{i-1}}{2}+\frac{p_{i+1}+p_{i}}{2}\right)\right]=\sum_{i=1}^{N_{x}} \varepsilon \Delta x b_{i}\left(\frac{p_{i}+p_{i-1}}{2}+\frac{p_{i+1}+p_{i}}{2}\right) .
\end{aligned}
$$

Then, remarking that

$$
\begin{aligned}
& \sum_{i=1}^{N_{x}} p_{i}\left(p_{i}+p_{i-1}+p_{i+1}+p_{i}\right)=\sum_{i=1}^{N_{x}}\left(p_{i}^{2}+\frac{1}{2} p_{i}^{2}+p_{i} p_{i-1}+\frac{1}{2} p_{i-1}^{2}+\frac{1}{2} p_{i}^{2}+p_{i} p_{i+1}+\frac{1}{2} p_{i+1}^{2}-\frac{1}{2} p_{i-1}^{2}-\frac{1}{2} p_{i+1}^{2}\right) \\
& =\sum_{i=1}^{N_{x}}\left(p_{i}^{2}-\frac{1}{2} p_{i+1}^{2}-\frac{1}{2} p_{i-1}^{2}+\frac{1}{2}\left(p_{i}+p_{i+1}\right)^{2}+\frac{1}{2}\left(p_{i}+p_{i-1}\right)^{2}\right)=\frac{1}{2}\left(p_{1}^{2}-p_{0}^{2}+p_{N_{x}}^{2}-p_{N_{x}+1}^{2}\right)+\frac{1}{2} \sum_{i=1}^{N_{x}}\left(p_{i}+p_{i+1}\right)^{2}+\left(p_{i}+p_{i-1}\right)^{2},
\end{aligned}
$$

and by using the boundary conditions, we obtain

$$
\begin{aligned}
\frac{\Delta x \varepsilon}{(\gamma-1)} \sum_{i=1}^{N_{x}}\left(\frac{p_{i}+p_{i+1}}{2}\right)^{2}+\left(\frac{p_{i}+p_{i-1}}{2}\right)^{2}-\frac{\Delta t^{2}}{2 \Delta x}\left[\sum_{i=1}^{N_{x}} \tilde{h}_{i+1 / 2}\left(\frac{p_{i+2}+p_{i+1}}{2}-\frac{p_{i}+p_{i-1}}{2}\right)\left(\frac{p_{i}+p_{i-1}}{2}+\frac{p_{i+1}+p_{i}}{2}\right)\right. \\
\left.-\sum_{i=0}^{N_{x}-1} \tilde{h}_{i+1 / 2}\left(\frac{p_{i+2}+p_{i+1}}{2}-\frac{p_{i}+p_{i-1}}{2}\right)\left(\frac{p_{i+1}+p_{i}}{2}+\frac{p_{i+2}+p_{i+1}}{2}\right)\right]=\sum_{i=1}^{N_{x}} \varepsilon \Delta x b_{i}\left(\frac{p_{i}+p_{i-1}}{2}+\frac{p_{i+1}+p_{i}}{2}\right),
\end{aligned}
$$


and then

$$
\begin{aligned}
\frac{\Delta x \varepsilon}{(\gamma-1)} \sum_{i=1}^{N_{x}}\left(\frac{p_{i}+p_{i+1}}{2}\right)^{2}+ & \left(\frac{p_{i}+p_{i-1}}{2}\right)^{2}+\frac{\Delta t^{2}}{2 \Delta x}\left[\sum_{i=1}^{N_{x}-1} \tilde{h}_{i+1 / 2}\left(\frac{p_{i+2}+p_{i+1}}{2}-\frac{p_{i}+p_{i-1}}{2}\right)^{2}\right. \\
& -\frac{1}{4} \tilde{h}_{N_{x}+1 / 2}\left(p_{N_{x}+2}+p_{N_{x}+1}-p_{N_{x}}-p_{N_{x}-1}\right)\left(p_{N_{x}}+p_{N_{x}-1}+p_{N_{x}+1}+p_{N_{x}}\right) \\
& \left.+\frac{1}{4} \tilde{h}_{1 / 2}\left(p_{2}+p_{1}-p_{0}-p_{-1}\right)\left(p_{1}+p_{0}+p_{2}+p_{1}\right)\right]=\sum_{i=1}^{N_{x}} \varepsilon \Delta x b_{i}\left(\frac{p_{i}+p_{i-1}}{2}+\frac{p_{i+1}+p_{i}}{2}\right)
\end{aligned}
$$

Finally, using the boundary conditions and $a(b+c) \leq \frac{1}{2}\left(2 a^{2}+\frac{1}{2}(b+c)^{2}\right) \leq a^{2}+\frac{1}{2}\left(b^{2}+c^{2}\right)$, we obtain

$$
\begin{aligned}
\frac{\Delta x \varepsilon}{(\gamma-1)} \sum_{i=1}^{N_{x}}\left(\frac{p_{i}+p_{i+1}}{2}\right)^{2}+\left(\frac{p_{i}+p_{i-1}}{2}\right)^{2}+\frac{\Delta t^{2}}{2 \Delta x} \sum_{i=1}^{N_{x}-1} \tilde{h}_{i+1 / 2}\left(\frac{p_{i+2}+p_{i+1}}{2}-\frac{p_{i}+p_{i-1}}{2}\right)^{2} & \\
& \leq \Delta x \varepsilon(\gamma-1) \sum_{i=1}^{N_{x}}\left|b_{i}\right|^{2}+\frac{\Delta x \varepsilon}{2(\gamma-1)} \sum_{i=1}^{N_{x}}\left(\frac{p_{i}+p_{i+1}}{2}\right)^{2}+\left(\frac{p_{i}+p_{i-1}}{2}\right)^{2},
\end{aligned}
$$

Which is (51). Now, if $b_{i}=0$ for all $i=0, \cdots N_{x}$ and if $\tilde{h}_{i+1 / 2} \geq 0$ for all $i=1, \cdots, N_{x}-1$, we obtain $p_{i}+p_{i+1}=0$ for all $i=0, \cdots N_{x}$. But $p_{0}=p_{1}$ then $p_{0}=p_{1}=0$ and so $p_{i}=0$ for all $i=0, \cdots, N_{x}+2$ and thus the system is well-posed.

The system (49)-(50) can be written in compact matrix form as

$$
\mathbf{T}^{r} \boldsymbol{p}^{n+1, r+1}=\varepsilon \Delta x \boldsymbol{b}^{r},
$$

with $\boldsymbol{p}^{n+1, r+1}=\left(p_{1}^{n+1, r+1}, \ldots, p_{N_{x}}^{n+1, r+1}\right)$ representing the vector of unknown pressures, and $\boldsymbol{b}^{r}=\left(b_{1}^{r}, \ldots, b_{N_{x}}^{r}\right)$ the right hand side entries given by (50). The system matrix $\mathbf{T}^{r}$ involves only positive quantities, namely pressure, enthalpy, time step and mesh size and has a pentadiagonal form. In particular, if $\varepsilon=0$, system (49) admits a constant in space pressure field which precise value depends on the chosen boundary conditions. This implies that the enthalpy is also constant in space. These two conditions finally give the incompressibility condition, i.e. $\partial_{x} u=0$, and, the asymptotically consistency with the incompressible limit also at the fully discrete level. The system matrix $\mathbf{T}^{r}$ is not proved to be symmetric, thus the pressure system is solved relying on the GMRES algorithm [85].

The initialization of all quantities defined at the first Picard iteration $(r=1)$ are taken as

$$
p_{i}^{n+1,1}=(\gamma-1) E_{i}^{n}-k_{i}^{n}, \quad q_{i}^{n+1,1}=F^{e}\left[q_{i}^{n}\right], \quad k_{i}^{n+1,1}=F^{e}\left[(\rho E)_{i}^{n}\right]-e_{i}^{n} .
$$

Thus, the iterative Picard algorithm and the time evolution of the solution work in the following way. Equation (52) provides a new pressure $p_{i}^{n+1, r+1}$, then the momentum equation (44) provides a new value for $q_{i}^{n+1, r+1}$

$$
q_{i}^{n+1, r+1}=F^{e}\left[(\rho u)_{i}^{n}\right]-\frac{1}{2} \frac{\Delta t}{\Delta x}\left(p_{i+1}^{n+1, r+1}-p_{i-1}^{n+1, r+1}\right),
$$

from which we can update the enthalpy (46) and the kinetic energy (48). Typically, from our numerical experiments, a total number of $R_{p}=2$ iterations are sufficient to obtain convergence of the nonlinear system (49)-(50). Therefore, after $R_{p}$ Picard iterations, we set:

$$
p_{i}^{n+1}=p_{i}^{n+1, R_{p}}, \quad q_{i}^{n+1}=q_{i}^{n+1, R_{p}} .
$$

Once the solution at time index $(n+1)$ in terms of pressure, enthalpy and momentum is found, then the total energy is updated thanks to the conservative equation (45). This concludes one time step of the proposed scheme.

Remark 1. In cases of high Mach number flows, additional stability can be required. If this is the case, the centered discretization (44) employed for the pressure field can be supplemented with a numerical dissipation as done for the explicit operator $F\left[q_{i}^{n}\right]$ in (42), thus leading to a modified scheme for the momentum equation. This reads

$$
\bar{q}_{i}^{n+1}=q_{i}^{n+1}-\frac{1}{2} s_{i+1 / 2}\left(p_{i+1}^{n+1}-p_{i}^{n+1}\right)-\frac{1}{2} s_{i-1 / 2}\left(p_{i}^{n+1}-p_{i-1}^{n+1}\right),
$$


where

$$
s_{i+1 / 2}=\left(\left|u_{i+1 / 2}^{n}\right|+\max \left(c_{i+1}^{n}, c_{i}^{n}\right)\right) \frac{1}{\gamma-1}, \quad s_{i-1 / 2}=\left(\left|u_{i-1 / 2}^{n}\right|+\max \left(c_{i}^{n}, c_{i-1}^{n}\right)\right) \frac{1}{\gamma-1} .
$$

The choice of the numerical disspation in (57) is different from the one proposed in [95] and it makes use of a Rusanovtype scheme that can be very general and allows for applications to different systems of governing equations, thus not being limited only to the Euler equations [37].

\subsection{Exact preservation of pressure and velocity across a contact discontinuity}

In this part, we discuss the ability of our novel scheme to preserve a class of exact solutions of the compressible Euler equation. In particular, we study the capability of the fully discrete scheme to maintain a pressure equilibrium across a contact wave. This condition has been studied in [5], for instance, and it consists in verifying that a constant pressure and a constant velocity field are preserved through a discontinuity in the fluid density during the time evolution of the solution. To that aim, let us consider the following initial conditions for all $i=1, \ldots N_{x}$ :

$$
\rho_{i}(t=0)=\rho_{i}^{0}=\left\{\begin{array}{ll}
\rho_{L} & x \leq x_{D} \\
\rho_{R} & x>x_{D}
\end{array}, \quad u_{i}(t=0)=u_{i}^{0}=u_{0}, \quad p_{i}(t=0)=p_{i}^{0}=p_{0}, \quad i=1, \ldots N_{x},\right.
$$

with $x_{D}$ representing a given position inside the domain and $\rho_{L} \neq \rho_{R}$ being non-negative real numbers. In this case, one has for the first time step

$$
\begin{aligned}
F^{e}\left[\rho_{i}^{0}\right] & =\rho_{i}^{0}-\frac{\Delta t}{\Delta x}\left(f_{i+1 / 2}^{\rho, 0}-f_{i-1 / 2}^{\rho, 0}\right)=\rho_{i}^{1}, \\
F^{e}\left[q_{i}^{0}\right] & =q_{i}^{0}-\frac{\Delta t}{\Delta x}\left(f_{i+1 / 2}^{q, 0}-f_{i-1 / 2}^{q, 0}\right)=\rho_{i}^{0} u_{0}-\frac{\Delta t}{\Delta x} u_{0}\left(f_{i+1 / 2}^{\rho, 0}-f_{i-1 / 2}^{\rho, 0}\right)=\rho_{i}^{1} u_{0}, \\
F^{e}\left[E_{i}^{0}\right] & =\underbrace{e_{i}^{0}+k_{i}^{0}}_{=E_{i}^{0}}-\frac{\Delta t}{\Delta x} \frac{\varepsilon u_{0}^{2}}{2}\left(f_{i+1 / 2}^{\rho, 0}-f_{i-1 / 2}^{\rho, 0}\right)=e_{i}^{0}+\rho_{i}^{0} \frac{\varepsilon u_{0}^{2}}{2}-\frac{\Delta t}{\Delta x} \frac{u_{0}^{2}}{2}\left(f_{i+1 / 2}^{\rho, 0}-f_{i-1 / 2}^{\rho, 0}\right)=\frac{p_{0}}{\gamma-1}+\rho_{i}^{1} \frac{\varepsilon u_{0}^{2}}{2} .
\end{aligned}
$$

Now, the pressure system (49) has to be solved for the first time step. We recall that the initial condition for the pressure (53) at the first Picard iteration $r=1$ leads to $p_{i}^{n+1,1}=p_{0}$, thus the interface enthalpies reduce to

$$
\tilde{h}_{i+1 / 2}^{n+1,1}=\left(2 p_{0} \frac{\gamma}{\gamma-1}\right)\left(\rho_{i}^{1}+\rho_{i+1}^{1}\right)^{-1}, \quad \tilde{h}_{i-1 / 2}^{n+1,1}=\left(2 p_{0} \frac{\gamma}{\gamma-1}\right)\left(\rho_{i}^{1}+\rho_{i-1}^{1}\right)^{-1} .
$$

Now the right hand side of the pressure system (49) becomes

$$
\varepsilon \Delta x b_{i}^{1}=\varepsilon \Delta x p_{0},
$$

therefore, thanks to (59)-(61), choosing $p^{1}=_{0}$ one obtains for $r=1$

$$
\frac{\varepsilon \Delta x}{\gamma-1} p_{0}=\varepsilon \Delta x \frac{p_{0}}{\gamma-1}-\varepsilon \frac{\varepsilon \Delta t}{2} \cdot 0,
$$

this means that the equations (44) and (45) are solved by $p_{i}^{n+1}=p_{0}$ and $u_{i}^{n+1}=u_{0}$ thus guaranteeing the preservation of the exact solution. Indeed, under the above hypothesis we get from (44)

$$
q_{i}^{1}=F^{e}\left[q_{i}^{0}\right]-\frac{\Delta t}{2 \varepsilon \Delta x}\left(p_{i+1}^{1}-p_{i-1}^{1}\right)=\rho_{i}^{1} u_{0}, \quad i=1, \ldots N_{x},
$$

and then by dividing the momentum $q_{i}^{1}$ at time level 1 by the density $\rho_{i}^{1}$ from (60) one obtains

$$
u_{i}^{1}=\frac{q_{i}^{1}}{\rho_{i}^{1}}=\frac{\rho_{i}^{1} u_{0}}{\rho_{i}^{1}}=u_{0}, \quad i=1, \ldots N_{x} .
$$


Substituting this result into the energy equation (45) leads to

$$
E_{i}^{1}=\frac{p_{0}}{\gamma-1}+\varepsilon \rho_{i}^{1} \frac{u_{0}^{2}}{2}-\frac{\Delta t}{2 \Delta x} \frac{\gamma u_{0}}{\gamma-1} \frac{\left(p_{i+1}^{1}-p_{i-1}^{1}\right)}{\varepsilon}=\frac{p_{0}}{\gamma-1}+\varepsilon \rho_{i}^{1} \frac{u_{0}^{2}}{2}=\frac{p_{i}^{1}}{\gamma-1}+\varepsilon \rho_{i}^{1} \frac{u_{0}^{2}}{2},
$$

which proves that $p_{i}^{1}=p_{0}$ and $u_{i}^{1}=u_{0}$ with $\rho_{i}^{1}=\rho_{i}^{0}-\frac{\Delta t}{\Delta x}\left(f_{i+1 / 2}^{\rho, 0}-f_{i-1 / 2}^{\rho, 0}\right)$ are a solution of the proposed numerical scheme corresponding to a shift of the density profile at speed $u_{0}$. Finally, being the structure of the solution preserved from time index $n=0$ to $n=1$, then the solution remains preserved for all $n \geq 1$. Numerical evidences of this property are reported in section 5.2, where a moving contact wave at low Mach number is shifted in time exactly preserving a constant pressure and velocity background field.

\subsection{Multi-dimensions extension}

In this part, we discuss the multi-dimensional extension of the previously introduced numerical scheme. The computational domain $\Omega=\left[x_{\min }, x_{\max }\right] \times\left[y_{\min }, y_{\max }\right] \times\left[z_{\min }, z_{\max }\right]$ is paved with $N_{x} \times N_{y} \times N_{z}$ uniform cells of size $\Delta x \times \Delta y \times \Delta z$. A cell is labeled by three indices ${ }_{i, j, k}$, namely one for each direction, while a face is referred to as $i+1 / 2, j, k, i, j+1 / 2, k$ or $i, j, k+1 / 2$ and, any unit normal vector to a face is denoted by $\boldsymbol{n}$. The cell center is located at point $\boldsymbol{x}_{i, j, k}=\left(x_{i}, y_{j}, z_{k}\right)$ and a face center is at point $\boldsymbol{x}_{i+1 / 2, j, k}=\left(\frac{1}{2}\left(x_{i}+x_{i+1}\right), y_{j}, z_{k}\right)$. By definition we have the normal component of the velocity with respect to a face given by

$$
\boldsymbol{u} \cdot \boldsymbol{e}_{x}=u, \quad \boldsymbol{u} \cdot \boldsymbol{e}_{y}=v, \quad \boldsymbol{u} \cdot \boldsymbol{e}_{z}=w,
$$

where $\boldsymbol{e}$ are the canonical unit vectors, that is $\boldsymbol{e}_{x}=(1,0,0), \boldsymbol{e}_{y}=(0,1,0), \boldsymbol{e}_{z}=(0,0,1)$. The numerical method in $3 \mathrm{D}$ follows the same path as its one dimensional version. Any generic cell-centered quantity $m_{i, j, k}$ is advected by a combination of an explicit and implicit flux. The explicit part reads

$$
F^{e}\left[m_{i, j, k}^{n}\right]=m_{i, j, k}^{n}-\frac{\Delta t}{\Delta x}\left(f_{i+1 / 2, j, k}^{m}-f_{i-1 / 2, j, k}^{m}\right)-\frac{\Delta t}{\Delta y}\left(g_{i, j+1 / 2, k}^{m}-g_{i, j-1 / 2, k}^{m}\right)-\frac{\Delta t}{\Delta z}\left(\omega_{i, j, k+1 / 2}^{m}-\omega_{i, j, k-1 / 2}^{m}\right),
$$

where the numerical fluxes are given by

$$
\begin{aligned}
f_{i+1 / 2, j, k}^{m} & =\frac{1}{2}\left(f\left(m_{i+1, j, k}^{n}\right)+f\left(m_{i, j, k}^{n}\right)\right)-\frac{1}{2}\left|u_{i+1 / 2, j, k}^{\max }\right|\left(m_{i+1, j, k}^{n}-m_{i, j, k}^{n}\right), \\
g_{i, j+1 / 2, k}^{m} & =\frac{1}{2}\left(g\left(m_{i, j+1, k}^{n}\right)+g\left(m_{i, j, k}^{n}\right)\right)-\frac{1}{2}\left|v_{i, j+1 / 2, k}^{\max }\right|\left(m_{i, j+1, k}^{n}-m_{i, j, k}^{n}\right), \\
\omega_{i, j, k+1 / 2}^{m} & =\frac{1}{2}\left(\omega\left(m_{i, j, k+1}^{n}\right)+\omega\left(m_{i, j, k}^{n}\right)\right)-\frac{1}{2}\left|w_{i, j, k+1 / 2}^{\max }\right|\left(m_{i, j, k+1}^{n}-m_{i, j, k}^{n}\right),
\end{aligned}
$$

with the speeds at the interfaces

$$
\left|u_{i+1 / 2, j, k}^{\max }\right|=\max \left(\left|u_{i+1, j, k}^{n}\right|,\left|u_{i, j, k}^{n}\right|\right), \quad\left|v_{i, j+1 / 2, k}^{\max }\right|=\max \left(\left|v_{i, j+1, k}^{n}\right|,\left|v_{i, j, k}^{n}\right|\right), \quad\left|w_{i, j, k+1 / 2}^{\max }\right|=\max \left(\left|w_{i, j, k+1}^{n}\right|,\left|w_{i, j, k}^{n}\right|\right) \text {. }
$$

The density $\rho_{i, j, k}^{n}$ is then computed as

$$
\rho_{i, j, k}^{n+1}=F^{e}\left[\rho_{i, j, k}^{n}\right],
$$

while the three components of the momentum equation satisfy

$$
\begin{aligned}
(\rho u)_{i, j, k}^{n+1} & =F^{e}\left[(\rho u)_{i, j, k}^{n}\right]-\frac{1}{2} \frac{\Delta t}{\varepsilon \Delta x}\left(p_{i+1, j, k}^{n+1}-p_{i-1, j, k}^{n+1}\right), \\
(\rho v)_{i, j, k}^{n+1} & =F^{e}\left[(\rho v)_{i, j, k}^{n}\right]-\frac{1}{2} \frac{\Delta t}{\varepsilon \Delta y}\left(p_{i, j+1, k}^{n+1}-p_{i, j-1, k}^{n+1}\right), \\
(\rho w)_{i, j, k}^{n+1} & =F^{e}\left[(\rho w)_{i, j, k}^{n}\right]-\frac{1}{2} \frac{\Delta t}{\varepsilon \Delta z}\left(p_{i, j, k+1}^{n+1}-p_{i, j, k-1}^{n+1}\right),
\end{aligned}
$$


where one notices that, as for the one dimensional case, the pressure is taken implicitly using a Rusanov flux without adding any numerical viscosity. The energy equation is expressed by

$$
\begin{aligned}
E_{i, j, k}^{n+1} & =F^{e}\left[E_{i, j, k}^{n}\right] \\
& \left.\left.-\frac{1}{2} \frac{\Delta t}{\Delta x}\left(\tilde{h}_{i+1 / 2, j, k}^{n+1}(\rho u)_{i+1, j, k}^{n+1}+(\rho u)_{i, j, k}^{n+1}\right)-\tilde{h}_{i-1 / 2, j, k}^{n+1}(\rho u)_{i, j, k}^{n+1}+(\rho u)_{i-1, j, k}^{n+1}\right)\right) \\
& \left.-\frac{1}{2} \frac{\Delta t}{\Delta y}\left(\tilde{h}_{i, j+1 / 2, k}^{n+1}(\rho v)_{i, j+1, k}^{n+1}+(\rho v)_{i, j, k}^{n+1}\right)-\tilde{h}_{i, j-1 / 2, k}^{n+1}\left((\rho v)_{i, j, k}^{n+1}+(\rho v)_{i, j-1, k}^{n+1}\right)\right) \\
& -\frac{1}{2} \frac{\Delta t}{\Delta z}\left(\tilde{h}_{i, j, k+1 / 2}^{n+1}\left((\rho w)_{i, j, k+1}^{n+1}+(\rho w)_{i, j, k}^{n+1}\right)-\tilde{h}_{i, j, k-1 / 2}^{n+1}(\rho w)_{i, j, k}^{n+1}+(\rho w)_{i, j, k-1}^{n+1}\right) .
\end{aligned}
$$

Then, by writing the total energy $E_{i, j, k}^{n+1}$ as the sum of internal $e_{i, j, k}^{n+1}$ and kinetic energy $k_{i, j, k}^{n+1}$, given by

$$
e_{i, j, k}^{n+1}=\frac{p_{i, j, k}^{n+1}}{\gamma-1}, \quad k_{i, j, k}^{n+1}=\frac{1}{2} \rho_{i, j, k}^{n+1}\left[\left(\frac{(\rho u)_{i, j, k}^{n+1}}{\rho_{i, j, k}^{n+1}}\right)^{2}+\left(\frac{(\rho v)_{i, j, k}^{n+1}}{\rho_{i, j, k}^{n+1}}\right)^{2}+\left(\frac{(\rho w)_{i, j, k}^{n+1}}{\rho_{i, j, k}^{n+1}}\right)^{2}\right],
$$

one recasts the energy equation by substituting the momentum equations (71)-(72)-(73) into (74) yielding

$$
\begin{aligned}
\frac{p_{i, j, k}^{n+1}}{\gamma-1} & =F^{e}\left[E_{i, j, k}^{n}\right]-k_{i, j, k}^{n+1} \\
& -\frac{1}{2} \frac{\Delta t}{\Delta x} \tilde{h}_{i+1 / 2, j, k}^{n+1}\left[\left(F^{e}\left[(\rho)_{i+1, j, k}^{n}\right]+F^{e}\left[(\rho)_{i, j, k}^{n}\right]\right)-\frac{1}{2} \frac{\Delta t}{\varepsilon \Delta x}\left(p_{i+2, j, k}^{n+1}-p_{i, j, k}^{n+1}+p_{i+1, j, k}^{n+1}-p_{i-1, j, k}^{n+1}\right)\right] \\
& +\frac{1}{2} \frac{\Delta t}{\Delta x} \tilde{h}_{i-1 / 2, j, k}^{n+1}\left[\left(F^{e}\left[(\rho u)_{i, j, k}^{n}\right]+F^{e}\left[(\rho u)_{i-1, j, k}^{n}\right]\right)-\frac{1}{2} \frac{\Delta t}{\varepsilon \Delta x}\left(p_{i+1, j, k}^{n+1}-p_{i-1, j, k}^{n+1}+p_{i, j, k}^{n+1}-p_{i-2, j, k}^{n+1}\right)\right] \\
& -\frac{1}{2} \frac{\Delta t}{\Delta y} \tilde{h}_{i, j+1 / 2, k}^{n+1}\left[\left(F^{e}\left[(\rho v)_{i, j+1, k}^{n}\right]+F^{e}\left[(\rho v)_{i, j, k}^{n}\right]\right)-\frac{1}{2} \frac{\Delta t}{\varepsilon \Delta y}\left(p_{i, j+2, k}^{n+1}-p_{i, j, k}^{n+1}+p_{i, j+1, k}^{n+1}-p_{i, j-1, k}^{n+1}\right)\right] \\
& +\frac{1}{2} \frac{\Delta t}{\Delta y} \tilde{h}_{i, j-1 / 2, k}^{n+1}\left[\left(F^{e}\left[(\rho v)_{i, j, k}^{n}\right]+F^{e}\left[(\rho v)_{i, j-1, k}^{n}\right]\right)-\frac{1}{2} \frac{\Delta t}{\varepsilon \Delta y}\left(p_{i, j+1, k}^{n+1}-p_{i, j-1, k}^{n+1}+p_{i, j, k}^{n+1}-p_{i, j-2, k}^{n+1}\right)\right] \\
& -\frac{1}{2} \frac{\Delta t}{\Delta z} \tilde{h}_{i, j, k+1 / 2}^{n+1}\left[\left(F^{e}\left[(\rho w)_{i, j, k+1}^{n}\right]+F^{e}\left[(\rho w)_{i, j, k}^{n}\right]\right)-\frac{1}{2} \frac{\Delta t}{\varepsilon \Delta z}\left(p_{i, j, k+2}^{n+1}-p_{i, j, k}^{n+1}+p_{i, j, k+1}^{n+1}-p_{i, j, k-1}^{n+1}\right)\right] \\
& +\frac{1}{2} \frac{\Delta t}{\Delta z} \tilde{h}_{i, j, k-1 / 2}^{n+1}\left[\left(F^{e}\left[(\rho w)_{i, j, k}^{n}\right]+F^{e}\left[(\rho w)_{i, j, k-1}^{n}\right]\right)-\frac{1}{2} \frac{\Delta t}{\varepsilon \Delta z}\left(p_{i, j, k+1}^{n+1}-p_{i, j, k-1}^{n+1}+p_{i, j, k}^{n+1}-p_{i, j, k-2}^{n+1}\right)\right] .
\end{aligned}
$$

As for the one dimensional case, in the above equation, the variables which are already known at time $t^{n+1}$ are the density $\rho_{i}^{n+1}$, because the continuity equation is solved explicitly, and the explicit operators $F\left[(\rho u)_{i, j, k}^{n}\right], F\left[(\rho v)_{i, j, k}^{n}\right]$ and $F\left[(\rho w)_{i, j, k}^{n}\right]$. The resulting system of equations is strongly nonlinear in the pressure unknown. To solve this system we rely again on a Picard algorithm to converge to the solution at time $t^{n+1}$ by selecting only the pressure terms as implicit in the iterative method. Following this strategy, the resulting nonlinear system is given by

$$
\begin{aligned}
& -\frac{1}{4} \frac{\Delta t^{2}}{\Delta x^{2}} \tilde{h}_{i+1 / 2, j, k}^{n+1, r} p_{i+2, j, k}^{n+1, r+1}-\frac{1}{4} \frac{\Delta t^{2}}{\Delta y^{2}} \tilde{h}_{i, j+1 / 2, k}^{n+1, r} p_{i, j+2, k}^{n+1, r+1}-\frac{1}{4} \frac{\Delta t^{2}}{\Delta z^{2}} \tilde{h}_{i, j, k+1 / 2}^{n+1, r} p_{i, j, k+2}^{n+1, r+1} \\
& -\frac{1}{4} \frac{\Delta t^{2}}{\Delta x^{2}}\left(\tilde{h}_{i+1 / 2, j, k}^{n+1, r}-\tilde{h}_{i-1 / 2, j, k}^{n+1, r}\right) p_{i+1, j, k}^{n+1, r+1}-\frac{1}{4} \frac{\Delta t^{2}}{\Delta y^{2}}\left(\tilde{h}_{i, j+1 / 2, k}^{n+1, r}-\tilde{h}_{i, j-1 / 2, k}^{n+1, r}\right) p_{i, j+1, k}^{n+1, r+1}-\frac{1}{4} \frac{\Delta t^{2}}{\Delta z^{2}}\left(\tilde{h}_{i, j, k+1 / 2}^{n+1, r}-\tilde{h}_{i, j, k-1 / 2}^{n+1, r}\right) p_{i, j, k+1}^{n+1, r+1} \\
& +\left(\frac{\varepsilon}{\gamma-1}+\frac{1}{4} \frac{\Delta t^{2}}{\Delta x^{2}}\left(\tilde{h}_{i+1 / 2, j, k}^{n+1, r}+\tilde{h}_{i-1 / 2, j, k}^{n+1, r}\right)+\frac{1}{4} \frac{\Delta t^{2}}{\Delta y^{2}}\left(\tilde{h}_{i, j+1 / 2, k}^{n+1, r}+\tilde{h}_{i, j-1 / 2, k}^{n+1, r}\right)+\frac{1}{4} \frac{\Delta t^{2}}{\Delta z^{2}}\left(\tilde{h}_{i, j, k+1 / 2}^{n+1, r}+\tilde{h}_{i, j, k-1 / 2}^{n+1, r}\right)\right) p_{i, j, k}^{n+1, r+1} \\
& +\frac{1}{4} \frac{\Delta t^{2}}{\Delta x^{2}}\left(\tilde{h}_{i+1 / 2, j, k}^{n+1, r}-\tilde{h}_{i-1 / 2, j, k}^{n+1, r}\right) p_{i-1, j, k}^{n+1, r+1}+\frac{1}{4} \frac{\Delta t^{2}}{\Delta y^{2}}\left(\tilde{h}_{i, j+1 / 2, k}^{n+1, r}-\tilde{h}_{i, j-1 / 2, k}^{n+1, r}\right) p_{i, j-1, k}^{n+1, r+1}+\frac{1}{4} \frac{\Delta t^{2}}{\Delta z^{2}}\left(\tilde{h}_{i, j, k+1 / 2}^{n+1, r}-\tilde{h}_{i, j, k-1 / 2}^{n+1, r}\right) p_{i, j, k-1}^{n+1, r+1} \\
& -\frac{1}{4} \frac{\Delta t^{2}}{\Delta x^{2}} \tilde{h}_{i-1 / 2, j, k}^{n+1, r} p_{i-2, j, k}^{n+1, r+1}-\frac{1}{4} \frac{\Delta t^{2}}{\Delta y^{2}} \tilde{h}_{i, j-1 / 2, k}^{n+1, r} p_{i, j-2, k}^{n+1, r+1}-\frac{1}{4} \frac{\Delta t^{2}}{\Delta z^{2}} \tilde{h}_{i, j, k-1 / 2}^{n+1, r} p_{i, j, k-2}^{n+1, r+1}=\varepsilon b_{i}^{r},
\end{aligned}
$$


where the right hand side reads

$$
\begin{aligned}
b_{i, j, k}^{r} & =\left(F\left[E_{i}^{n}\right]-k_{i}^{n+1, r}\right) \\
& -\frac{1}{2} \frac{\Delta t}{\Delta x}\left(\tilde{h}_{i+1 / 2, j, k}^{n+1, r} F^{e}\left[(\rho u)_{i+1, j, k}^{n}\right]+\left(\tilde{h}_{i+1 / 2, j, k}^{n+1, r}-\tilde{h}_{i-1 / 2, j, k}^{n+1, r}\right) F^{e}\left[(\rho u)_{i, j, k}^{n}\right]-\tilde{h}_{i-1 / 2, j, k}^{n+1, r} F^{e}\left[(\rho u)_{i-1, j, k}^{n}\right]\right), \\
& -\frac{1}{2} \frac{\Delta t}{\Delta y}\left(\tilde{h}_{i, j+1 / 2, k}^{n+1, r} F^{e}\left[(\rho v)_{i, j+1, k}^{n}\right]+\left(\tilde{h}_{i, j+1 / 2, k}^{n+1, r}-\tilde{h}_{i, j-1 / 2, k}^{n+1, r}\right) F^{e}\left[(\rho v)_{i, j, k}^{n}\right]-\tilde{h}_{i, j-1 / 2, k}^{n+1, r} F^{e}\left[(\rho v)_{i, j-1, k}^{n}\right]\right), \\
& -\frac{1}{2} \frac{\Delta t}{\Delta z}\left(\tilde{h}_{i, j, k+1 / 2}^{n+1, r} F^{e}\left[(\rho w)_{i, j, k+1}^{n}\right]+\left(\tilde{h}_{i, j, k+1 / 2}^{n+1, r}-\tilde{h}_{i, j, k-1 / 2}^{n+1, r}\right) F^{e}\left[(\rho w)_{i, j, k}^{n}\right]-\tilde{h}_{i, j, k-1 / 2}^{n+1, r} F^{e}\left[(\rho w)_{i, j, k-1}^{n}\right]\right) .
\end{aligned}
$$

As for the one dimensional case, the iterative system involves only positive quantities, namely pressure, enthalpy, time step and mesh size. In particular, if $\varepsilon=0$, system (76) admits a constant in space pressure field as solution which value depends on the imposed boundary conditions. This implies that the enthalpy is also constant in space. These two conditions finally give the incompressibility condition, i.e.

$$
\nabla_{\boldsymbol{x}} \cdot \boldsymbol{u}_{i, j, k}^{n+1}=\frac{u_{i+1, j, k}^{n+1}-u_{i-1, j, k}^{n+1}}{2 \Delta x}+\frac{v_{i, j+1, k}^{n+1}-v_{i, j-1, k}^{n+1}}{2 \Delta y}+\frac{w_{i, j, k+1}^{n+1}-w_{i, j, k-1}^{n+1}}{2 \Delta z}=0,
$$

and the asymptotically consistency with the incompressible limit at the fully discrete level. The initialization of all quantities defined at the first step of the Picard iterative solver $(r=1)$ directly follows what already presented for the one dimensional case, given by (53). Thus, the iterative Picard algorithm works in the following way: equation (76) provides a new pressure $p_{i, j, k}^{n+1, r+1}$, then the momentum equations (71)-(73) give a new value for $(\rho u)_{i, j, k}^{n+1, r+1},(\rho v)_{i, j, k}^{n+1, r+1}$, $(\rho w)_{i, j, k}^{n+1, r+1}$ from which we can update the enthalpy and the kinetic energy. Once the solution at time index $(n+1)$ in terms of pressure, enthalpy and momentum is found, then the total energy is updated thanks to equation (75). This concludes one time step of the proposed scheme.

Thanks to the semi-implicit discretization finally, the CFL condition is only dictated by the fluid velocity $\boldsymbol{u}$, and not by the sound speed $c$, hence

$$
\Delta t \leq \operatorname{CFL} \frac{\min (\Delta x, \Delta y, \Delta z)}{\max _{i, j, k}\left|2 \boldsymbol{u}_{i, j, k}^{n}\right|} .
$$

\section{A second order in time and space scheme for the Euler equations in all Mach regimes}

In this part we discuss how the first-order accurate scheme proposed in the previous section can be extended to a scheme with second order accuracy in time and space. We first discuss the time marching approach through a semidiscretization of the Euler equations (14) and we study its properties. In a second part, we give the details of the discretization of the space variables.

\subsection{A second-order in time scheme}

The second-order in time extension is based on an Implicit-Explicit (IMEX) Runge-Kutta approach [3, 75, 76, $32,8,33,9,6]$. In this work, to improve the time accuracy of our first order method, we use the second-order Ascher, Ruuth and Spiteri [3] tableaux denoted in the following by $\operatorname{ARS}(2,2,2)$, where the arguments in the acronym means that the explicit and the implicit schemes have both two stages (first and second number) and are second order accurate (third number), the same holds true for the global scheme. We recall that implicit methods of order higher than one for hyperbolic problems cannot be TVD [42] and this situation does not change when IMEX methods are employed [30]. In this work, as explained in the Introduction, we do not focus on this limit imposed on the high order schemes and we propose a second order scheme which, in principle, may not assure $L^{\infty}$ stability. Possible remedies are postponed to future investigations. The Butcher tableaux of the $\operatorname{ARS}(2,2,2)$ discretization are detailed in Table 1, where $\beta=1-\sqrt{2} / 2$ and $\alpha=1-1 /(2 \beta)$. Remarking that $\alpha=\beta-1$ and $1-\alpha=2-\beta$, the following scheme for the Euler equations (15) is obtained:

$$
\begin{aligned}
& \frac{\boldsymbol{Q}^{n, \star}-\boldsymbol{Q}^{n}}{\Delta t}+\beta \nabla_{\boldsymbol{x}} \cdot \mathcal{L}_{i}\left(\boldsymbol{Q}^{n, \star}\right)+\beta \nabla_{\boldsymbol{x}} \cdot \mathcal{L}_{e}\left(\boldsymbol{Q}^{n}\right)=0 \\
& \frac{\boldsymbol{Q}^{n+1}-\boldsymbol{Q}^{n}}{\Delta t}+(\beta-1) \nabla_{\boldsymbol{x}} \cdot \mathcal{L}_{e}\left(\boldsymbol{Q}^{n}\right)+(2-\beta) \nabla_{\boldsymbol{x}} \cdot \mathcal{L}_{e}\left(\boldsymbol{Q}^{n, \star}\right)+(1-\beta) \nabla_{\boldsymbol{x}} \cdot \mathcal{L}_{i}\left(\boldsymbol{Q}^{n, \star}\right)+\beta \nabla_{\boldsymbol{x}} \cdot \mathcal{L}_{i}\left(\boldsymbol{Q}^{n+1}\right)=0
\end{aligned}
$$




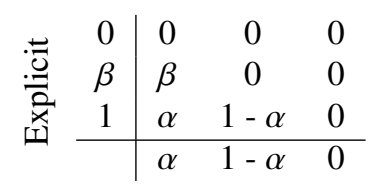

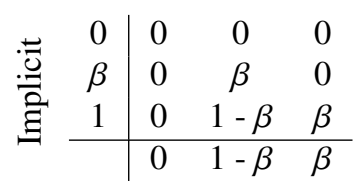

Table 1: Butcher tableaux for the $\operatorname{ARS}(2,2,2)$ time discretization. Left panel: explicit tableau. Right panel: implicit tableau. $\beta=1-\frac{\sqrt{2}}{2}, \alpha=\beta-1$.

The partition between $\mathcal{L}_{i}=\left(\boldsymbol{F}^{i m}, \boldsymbol{G}^{i m}, \boldsymbol{W}^{i m}\right)$, the implicit operator, and, $\mathcal{L}_{e}=\left(\boldsymbol{F}^{e x}, \boldsymbol{G}^{e x}, \boldsymbol{W}^{e x}\right)$, the explicit one is the same of the first order case. In the following, we furnish a formal proof that the scheme (80)-(81) provides a consistent and second order accurate discretization of the limit model (19)-(21) when $\varepsilon \rightarrow 0$.

Lemma 3. We denote by $\left(\rho_{\varepsilon}^{n}(\boldsymbol{x}), \boldsymbol{u}_{\varepsilon}^{n}(\boldsymbol{x}), E_{\varepsilon}^{n}(\boldsymbol{x})\right)_{n \geq 1}$ the solution of the semi discrete numerical scheme (80)-(81) with the boundary condition $\boldsymbol{u}^{n}(\boldsymbol{x}) \cdot \boldsymbol{v}(\boldsymbol{x})=0, \forall \boldsymbol{x} \in \partial \Omega, \forall n \geq 0$. Then, assuming that $\left(\rho_{\varepsilon}^{n}(\boldsymbol{x}), \boldsymbol{u}_{\varepsilon}^{n}(\boldsymbol{x}), E_{\varepsilon}^{n}(\boldsymbol{x})\right)_{n \geq 1}$ converge towards some finite values when $\varepsilon \rightarrow 0$, if the initial data are well prepared, the semi discrete numerical scheme is asymptotically consistent and accurate, i.e. in the limit $\varepsilon \rightarrow 0$ it becomes a consistent and second order in time discretization of system (19)-(21) from $n \geq 1$. Moreover, from $n \geq 2$, the scheme becomes a consistent and second order time discretization of the limit system (19)-(21) independently on the initial data.

Proof. We assume that, for all $n \geq 1$, all quantities $\rho_{\varepsilon}^{n}(\boldsymbol{x}), \boldsymbol{u}_{\varepsilon}^{n}(\boldsymbol{x}), p_{\varepsilon}^{n}(\boldsymbol{x})$ and $E_{\varepsilon}^{n}(\boldsymbol{x})$, solutions of (80)-(81), converge respectively towards some values $\rho^{n}(\boldsymbol{x}), \boldsymbol{u}^{n}(\boldsymbol{x}), p^{n}(\boldsymbol{x})$ and $E^{n}(\boldsymbol{x})$ when $\varepsilon$ tends to 0 . Under this hypothesis, multiplying by $\varepsilon$ the momentum equations of (80) and letting $\varepsilon$ tend to zero, gives $\nabla_{x} p^{n, \star}(\boldsymbol{x})=0$, i.e. the pressure at time level $(n, \star)$ is constant. From equation $(28)$, we have

$$
E_{\varepsilon}^{n, \star}(\boldsymbol{x})=e_{\varepsilon}^{n, \star}(\boldsymbol{x})+\frac{\varepsilon}{2} \rho_{\varepsilon}^{n, \star}(\boldsymbol{x})\left|\boldsymbol{u}_{\varepsilon}^{n, \star}(\boldsymbol{x})\right|^{2}=\frac{p_{\varepsilon}^{n, \star}(\boldsymbol{x})}{(\gamma-1)}+\frac{\varepsilon}{2} \rho_{\varepsilon}^{n, \star}(\boldsymbol{x})\left|\boldsymbol{u}_{\varepsilon}^{n, \star}(\boldsymbol{x})\right|^{2},
$$

which gives $E^{n, \star}(\boldsymbol{x})=p^{n, \star}(\boldsymbol{x}) /(\gamma-1)$ in the limit $\varepsilon \rightarrow 0$. Since the explicit part of the energy equation depends on the kinetic term which goes to zero in the limit, for all $x \in \Omega$ the limit yields

$$
E^{n, \star}(\boldsymbol{x})=E^{n}(\boldsymbol{x})-\beta \Delta t\left(e^{n, \star}(\boldsymbol{x})+p^{n, \star}(\boldsymbol{x})\right) \nabla_{\boldsymbol{x}} \cdot \boldsymbol{u}^{n, \star}(\boldsymbol{x}) .
$$

Thus, integrating this equation on the domain, using the Green formula and the boundary condition gives

$$
\begin{array}{r}
|\Omega| E^{n, \star}(\boldsymbol{x})=\int_{\Omega} E^{n}(\boldsymbol{x}) d \boldsymbol{x}-\beta \Delta t\left(e^{n}(\boldsymbol{x})+p^{n}(\boldsymbol{x})\right) \int_{\partial \Omega} \boldsymbol{u}^{n}(\boldsymbol{x}) \cdot \boldsymbol{v}(\boldsymbol{x}) d \sigma(\boldsymbol{x})+ \\
-\beta \Delta t\left(e^{n, \star}(\boldsymbol{x})+p^{n, \star}(\boldsymbol{x})\right) \int_{\partial \Omega} \boldsymbol{u}^{n, \star}(\boldsymbol{x}) \cdot \boldsymbol{v}(\boldsymbol{x}) d \sigma(\boldsymbol{x})=\int_{\Omega} E^{n}(\boldsymbol{x}) d \boldsymbol{x}
\end{array}
$$

which means that both pressure and energy are projected over constant states $\left(\tilde{p}^{0}, E^{0}\right)$ at the intermediate stage level $(n, \star)$ if initial data are well prepared, i.e. $E^{0}(\boldsymbol{x})=E^{0}$. Using again (82), gives under the hypothesis of consistent initial data $\nabla_{\boldsymbol{x}} \cdot \boldsymbol{u}^{n, \star}(\boldsymbol{x})=0$, and

$$
\frac{\left(\rho^{n, \star} \boldsymbol{u}^{n, \star}\right)(\boldsymbol{x})-\left(\rho^{n} \boldsymbol{u}^{n}\right)(\boldsymbol{x})}{\Delta t}+\beta \nabla_{\boldsymbol{x}} \cdot\left(\left(\rho^{n} \boldsymbol{u}^{n}\right)(\boldsymbol{x}) \otimes \boldsymbol{u}^{n}(\boldsymbol{x})\right)+\beta \nabla_{\boldsymbol{x}} p_{1}^{n, \star}(\boldsymbol{x})=0
$$

where we have assumed that the following limit for $\varepsilon \rightarrow 0$ exists

$$
\lim _{\varepsilon \rightarrow 0} \frac{1}{\varepsilon}\left(p_{\varepsilon}^{n, \star}-p^{n, \star}\right)=\lim _{\varepsilon \rightarrow 0} \frac{1}{\varepsilon}\left(p_{\varepsilon}^{n, \star}-\frac{1}{|\Omega|} \int_{\Omega} \vec{p}^{0}(\boldsymbol{x}) d \boldsymbol{x}\right)=p_{1}^{n, \star} .
$$

Otherwise, if initial data are not well prepared, the incompressibility condition is not in general verified. This means that for the first time step, the velocity field obeys to

$$
\nabla_{\boldsymbol{x}} \cdot \boldsymbol{u}^{0, \star}=-\frac{E^{0, \star}-E^{0}(\boldsymbol{x})}{\Delta t\left(e^{0, \star}+p^{0, \star}\right)} .
$$


We discuss now the second step of the Runge-Kutta method. We have again that multiplying by $\varepsilon$ the momentum equations of (81) and letting $\varepsilon$ tend to zero, gives $\nabla_{x} p^{n+1}(\boldsymbol{x})=0$, i.e. the pressure at time level $(n+1)$ is constant. The energy equation yields

$$
E^{n+1}(\boldsymbol{x})=E^{n}(\boldsymbol{x})-(1-\beta) \Delta t\left(e^{n, \star}(\boldsymbol{x})+p^{n, \star}(\boldsymbol{x})\right) \nabla_{\boldsymbol{x}} \cdot \boldsymbol{u}^{n, \star}(\boldsymbol{x})-\beta \Delta t\left(e^{n+1}(\boldsymbol{x})+p^{n+1}(\boldsymbol{x})\right) \nabla_{\boldsymbol{x}} \cdot \boldsymbol{u}^{n+1}(\boldsymbol{x}) .
$$

Now, by integration in space the above equation and using the boundary conditions, one ends with

$$
|\Omega| E^{n+1}(\boldsymbol{x})=\int_{\Omega} E^{n}(\boldsymbol{x}) d \boldsymbol{x},
$$

which means that the energy is projected over a constant state in the limit $\varepsilon \rightarrow 0$. On the other hand, the pressure field is given, in the hypothesis of existence of the $\operatorname{limit}_{\varepsilon \rightarrow 0} \frac{1}{\varepsilon}\left(p_{\varepsilon}^{n+1}-p^{n+1}\right)$, by

$$
\begin{gathered}
\frac{\left(\rho^{n+1} \boldsymbol{u}^{n+1}\right)(\boldsymbol{x})-\left(\rho^{n} \boldsymbol{u}^{n}\right)(\boldsymbol{x})}{\Delta t}+(\beta-1) \nabla_{\boldsymbol{x}} \cdot\left(\left(\rho^{n} \boldsymbol{u}^{n}\right)(\boldsymbol{x}) \otimes \boldsymbol{u}^{n}(\boldsymbol{x})\right)+ \\
+(2-\beta) \nabla_{\boldsymbol{x}} \cdot\left(\left(\rho^{n, \star} \boldsymbol{u}^{n, \star}\right)(\boldsymbol{x}) \otimes \boldsymbol{u}^{n, \star}(\boldsymbol{x})\right)+(1-\beta) \nabla_{\boldsymbol{x}} p_{1}^{n, \star}(\boldsymbol{x})+\beta \nabla_{\boldsymbol{x}} p_{1}^{n+1}(\boldsymbol{x})=0 .
\end{gathered}
$$

Finally, we have that the incompressibility constraint is satisfied if initial data are well prepared

$$
\nabla_{\boldsymbol{x}} \cdot \boldsymbol{u}^{n+1}=0, \quad \forall n \geq 1,
$$

while this holds true only for $n \geq 2$ for non well prepared initial data because

$$
\nabla_{\boldsymbol{x}} \cdot \boldsymbol{u}^{n+1}=-\frac{E^{n+1}-E^{n}}{\Delta t\left(e^{n+1}+p^{n+1}\right)}=0, \quad n \geq 2,
$$

and

$$
\nabla_{\boldsymbol{x}} \cdot \boldsymbol{u}^{1}=-\frac{E^{1}-E^{0}(\boldsymbol{x})}{\Delta t\left(e^{1}+p^{1}\right)} \neq 0,
$$

for the first time step, since energy is not necessarily constant at time $t=0$.

\subsection{Second-order in space}

In order to extend the space accuracy to a second order, we use a piecewise linear reconstruction of the conserved variables supplemented with classical minmod limiter to avoid the development of spurious oscillations. For a cell $c:=(i, j, k)$, the linear reconstruction polynomial $\mathbf{w}_{h}(x, y, z)$ writes

$$
\mathbf{w}_{h, c}(x, y, z)=\boldsymbol{Q}_{c}+\left(x-x_{c}\right) \boldsymbol{\alpha}_{x, c}+\left(x-y_{c}\right) \boldsymbol{\alpha}_{y, c}+\left(z-z_{c}\right) \boldsymbol{\alpha}_{z, c}
$$

where $\boldsymbol{Q}_{c}=\left(\rho_{c}, q_{c}, E_{c}\right)$ is the vector of conserved mean values, $\mathbf{x}_{c}:=\left(x_{c}, y_{c}, z_{c}\right)$ denotes the position of the centroid of cell $c$ and $\boldsymbol{\Sigma}_{c}:=\left(\boldsymbol{\alpha}_{x, c}, \boldsymbol{\alpha}_{y, c}, \boldsymbol{\alpha}_{z, c}\right)$ is the vector of slopes in $x, y$ and $z$ direction. Due to the fact that we use a threedimensional Cartesian grid, the reconstruction is efficiently carried out dimension-by-dimension. Thus, let us consider the $x$ direction with cell $c$ and its direct Neumann neighbors $L:=(i-1, j, k)$ and $R:=(i+1, j, k)$, which are nothing but the so-called reconstruction stencil $\mathcal{S}_{c}:=(L, c, R)$. The unknown reconstruction coefficient $\boldsymbol{\alpha}_{x, c}$ is computed by applying a classical minmod limiter to the left $\boldsymbol{\alpha}_{x, L}$ and right $\boldsymbol{\alpha}_{x, R}$ slopes, that is

$$
\boldsymbol{\alpha}_{x, c}=\left\{\begin{array}{lll}
0 & \text { if } & \boldsymbol{\alpha}_{x, L} \boldsymbol{\alpha}_{x, R} \leq 0 \\
\boldsymbol{\alpha}_{x, L} & \text { if } & \left|\boldsymbol{\alpha}_{x, L}\right| \leq\left|\boldsymbol{\alpha}_{x, R}\right| \\
\boldsymbol{\alpha}_{x, R} & \text { if } & \left|\boldsymbol{\alpha}_{x, L}\right|>\left|\boldsymbol{\alpha}_{x, R}\right|
\end{array}\right.
$$

where

$$
\boldsymbol{\alpha}_{x, L}=\frac{\boldsymbol{Q}_{c}-\boldsymbol{Q}_{L}}{\Delta x}, \quad \boldsymbol{\alpha}_{x, R}=\frac{\boldsymbol{Q}_{c}-\boldsymbol{Q}_{L}}{\Delta x} .
$$

Notice that the slopes are evaluated in (93) for each conserved variable of the Euler system contained in $\boldsymbol{Q}_{c}$. The same procedure applies for $y$ and $z$ direction independently, hence obtaining explicit values for all the reconstruction coefficients $\boldsymbol{\Sigma}_{c} \in \mathbb{R}$ in (91). The reconstruction is carried out only for all terms evaluated at time $t^{n}$ because it is needed to feed the numerical fluxes of the explicit operators detailed in section 3.2. Notice that all implicit terms are discretized with a centered second-order operator, which does not need any reconstruction procedure. 


\section{Numerical experiments}

In this section, we perform some numerical test cases which aim at demonstrating the accuracy and the robustness of our numerical method. A wide range of Mach numbers are considered, enhancing the capability of the pressure based implicit-explicit (P-IMEX) schemes to deal with strong shocks as well as smooth solutions. Specifically, we consider an isentropic vortex flow for studying the convergence, a set of Riemann problems with shock waves, including one Riemann problem that gives evidences about the asymptotic preserving property detailed in sections 3.3 and 4.1. Finally, the Gresho vortex test problem is run at different Mach numbers and an explosion problem is performed using approximately 6 million cells. Comparison with a fully explicit method is also proposed. If not specified, in all the simulations shown hereafter, the CFL number is set to $C F L=0.95$ in (40) and a total number of $R_{p}=2$ has been used for the iterative solver (49). Finally, the rescaled parameter is set to $\varepsilon=1$ if not stated otherwise.

\subsection{Isentropic vortex}

The isentropic vortex problem was initially introduced in a two-dimensional setting [51] to test the accuracy of numerical methods since the analytical solution is known and regular. On the computational domain $\Omega=[-5 ; 5]^{3}$, the flow is characterized by $\rho_{\infty}=1.0, u_{\infty}=1.0, v_{\infty}=1.0, w_{\infty}=0.0, p_{\infty}=1.0$, with a normalized temperature $T_{\infty}^{*}=1.0$. A vortex centred on the $z$-axis line at $\left(x_{c}, y_{c}\right)=(0,0)$ supplements the background gas at time $t=0$ with the following conditions:

$$
\rho=\rho_{\infty}+\delta \rho, \quad u=u_{\infty}+\delta u, \quad v=v_{\infty}+\delta v, \quad w=w_{\infty}, \quad T^{*}=T_{\infty}^{*}+\delta T^{*},
$$

where the perturbations for density and pressure read

$$
\delta \rho=\left(1+\delta T^{*}\right)^{\frac{1}{\gamma-1}}-1, \quad \delta p=\left(1+\delta T^{*}\right)^{\frac{\gamma}{\gamma-1}}-1,
$$

with the temperature fluctuation $\delta T^{*}=-\frac{(\gamma-1) \lambda^{2}}{8 \gamma \pi^{2}} e^{1-r^{2}}$. According to [51], the vortex strength is $\lambda=5$ and the adiabatic index is set to $\gamma=1.4$, while the velocity field is affected by the following perturbations:

$$
\left(\begin{array}{c}
\delta u \\
\delta v
\end{array}\right)=\frac{\lambda}{2 \pi} e^{\frac{1-r^{2}}{2}}\left(\begin{array}{r}
-y^{\prime} \\
x^{\prime}
\end{array}\right)
$$

with $r=\sqrt{x^{\prime 2}+y^{\prime 2}}$ and $x^{\prime}=x-x_{c}, y^{\prime}=y-y_{c}$. Periodic boundary conditions are prescribed and the final time is $t_{\text {final }}=1$. The simulation has been run on a sequence of four successively refined Cartesian meshes on the $x y$ plane with characteristic mesh size $h(\Omega)=L_{\text {char }} / N_{x}$ with $L_{c h a r}=10$. The exact solution is the time-shifted initial condition at speed $\left(u_{\infty}, v_{\infty}\right)$, thus we compute the errors in $L^{2}$ norm for the density, velocity and pressure variables, and the numerical order of convergence can be deduced for both space and time discretization, simultaneously. Tables 2 and 3 report the data obtained for the first order and second order P-IMEX schemes, respectively. Comparison against the corresponding explicit algorithms are shown as well. The explicit methods are based on first order Godunov finite volume schemes and on MUSCL TVD algorithms for the second order case. Here, the spatial reconstruction is carried out as described in section 4.2, while the time evolution is performed according to the MUSCL scheme [98] and numerical fluxes are evaluated relying on the approximate Rusanov solver. The formal order of accuracy is achieved and the P-IMEX schemes are proven to be more accurate for order $O(2)$.

\subsection{Riemann problems in $1 D$}

In this section, we want to test the proposed numerical method against some classical Riemann problems whose initial states are summarized in Table 4. For all the tests we use a 3D grid made of $N_{x}=200, N_{y}=20$ and $N_{z}=5$ cells on the computational domain $\Omega=[0 ; 1] \times[0 ; 0.1] \times[0 ; 0.1]$. Periodic boundaries are set in $y$ - and $z$ - direction, while Dirichlet conditions based on the initial state are imposed at $x=0$ and $x=1$. The Riemann problem RP1 and RP2, known as Sod and Lax problems, represent two benchmarks in gas dynamics [94]. They both are constituted by a right-moving shock wave, a left-moving rarefaction fan and an intermediate contact discontinuity. The Mach number ranges from 0 to 1 for Sod problem and 0.17 to 0.87 for Lax. The results given by the P-IMEX schemes are depicted in Figures 2 and 3, respectively, where both first and second order simulations are considered. 
Table 2: Numerical convergence results for the compressible Euler equations using the first order P-IMEX scheme and comparison with the corresponding explicit method. The errors are measured in $L_{2}$ norm and refer to the variables $\rho$ (density), $u$ (horizontal velocity) and $p$ (pressure) at time $t=1$.

\begin{tabular}{c|cccccc}
\multicolumn{7}{c}{ P-IMEX $O 1$} \\
\hline$h(\Omega)$ & $\rho_{L_{2}}$ & $O(\rho)$ & $u_{L_{2}}$ & $O(u)$ & $p_{L_{2}}$ & $O(p)$ \\
\hline $5.00 \mathrm{E}-01$ & $3.949 \mathrm{E}-01$ & - & $1.149 \mathrm{E}-00$ & - & $8.043 \mathrm{E}-01$ & - \\
$3.82 \mathrm{E}-01$ & $2.847 \mathrm{E}-01$ & 1.2 & $8.140 \mathrm{E}-01$ & 1.3 & $5.884 \mathrm{E}-01$ & 1.2 \\
$3.15 \mathrm{E}-01$ & $2.230 \mathrm{E}-01$ & 1.3 & $6.308 \mathrm{E}-01$ & 1.3 & $4.638 \mathrm{E}-01$ & 1.2 \\
$2.71 \mathrm{E}-01$ & $1.834 \mathrm{E}-01$ & 1.3 & $5.152 \mathrm{E}-01$ & 1.4 & $3.827 \mathrm{E}-01$ & 1.3 \\
\hline
\end{tabular}

EXPLICIT $O 1$

\begin{tabular}{c|cccccc}
\hline$h(\Omega)$ & $\rho_{L_{2}}$ & $O(\rho)$ & $u_{L_{2}}$ & $O(u)$ & $p_{L_{2}}$ & $O(p)$ \\
\hline $5.00 \mathrm{E}-01$ & $9.132 \mathrm{E}-01$ & - & $2.139 \mathrm{E}-00$ & - & $1.393 \mathrm{E}-01$ & - \\
$3.82 \mathrm{E}-01$ & $6.983 \mathrm{E}-01$ & 1.0 & $1.601 \mathrm{E}-01$ & 1.1 & $1.082 \mathrm{E}-01$ & 0.9 \\
$3.15 \mathrm{E}-01$ & $5.634 \mathrm{E}-01$ & 1.1 & $1.277 \mathrm{E}-01$ & 1.2 & $8.801 \mathrm{E}-01$ & 1.1 \\
$2.71 \mathrm{E}-01$ & $4.717 \mathrm{E}-01$ & 1.2 & $1.062 \mathrm{E}-01$ & 1.2 & $7.403 \mathrm{E}-01$ & 1.2 \\
\hline
\end{tabular}

Table 3: Numerical convergence results for the compressible Euler equations using the second order P-IMEX scheme and comparison with the corresponding explicit method (MUSCL-TVD discretization). The errors are measured in $L_{2}$ norm and refer to the variables $\rho$ (density), $u$ (horizontal velocity) and $p$ (pressure) at time $t=1$.

P-IMEX O2

\begin{tabular}{c|cccccc}
\hline$h(\Omega)$ & $\rho_{L_{2}}$ & $O(\rho)$ & $u_{L_{2}}$ & $O(u)$ & $p_{L_{2}}$ & $O(p)$ \\
\hline $5.00 \mathrm{E}-01$ & $2.149 \mathrm{E}-01$ & - & $4.565 \mathrm{E}-01$ & - & $2.164 \mathrm{E}-01$ & - \\
$3.82 \mathrm{E}-01$ & $1.427 \mathrm{E}-01$ & 2.0 & $2.559 \mathrm{E}-01$ & 2.1 & $1.166 \mathrm{E}-01$ & 2.3 \\
$3.15 \mathrm{E}-01$ & $9.161 \mathrm{E}-02$ & 2.3 & $1.673 \mathrm{E}-01$ & 2.2 & $7.167 \mathrm{E}-02$ & 2.5 \\
$2.71 \mathrm{E}-01$ & $6.578 \mathrm{E}-02$ & 2.2 & $1.184 \mathrm{E}-01$ & 2.3 & $4.956 \mathrm{E}-02$ & 2.5 \\
\hline
\end{tabular}

EXPLICIT O2

\begin{tabular}{c|cccccc}
\hline$h(\Omega)$ & $\rho_{L_{2}}$ & $O(\rho)$ & $u_{L_{2}}$ & $O(u)$ & $p_{L_{2}}$ & $O(p)$ \\
\hline $5.00 \mathrm{E}-01$ & $2.873 \mathrm{E}-01$ & - & $6.620 \mathrm{E}-00$ & - & $4.220 \mathrm{E}-01$ & - \\
$3.82 \mathrm{E}-01$ & $1.673 \mathrm{E}-01$ & 2.0 & $3.966 \mathrm{E}-01$ & 1.9 & $2.126 \mathrm{E}-01$ & 2.5 \\
$3.15 \mathrm{E}-01$ & $1.108 \mathrm{E}-01$ & 2.1 & $2.621 \mathrm{E}-01$ & 2.2 & $1.303 \mathrm{E}-01$ & 2.6 \\
$2.71 \mathrm{E}-01$ & $8.121 \mathrm{E}-02$ & 2.1 & $1.848 \mathrm{E}-01$ & 2.4 & $8.840 \mathrm{E}-02$ & 2.6 \\
\hline
\end{tabular}

Table 4: Initialization of $1 \mathrm{~d}$ test cases.

\begin{tabular}{cc|c|ccc|ccc|ccc}
\hline Name & $\mathrm{RP} \#$ & $t_{\text {final }}$ & $x_{L}$ & $x_{R}$ & $x_{d}$ & $\rho_{L}$ & $u_{L}$ & $p_{L}$ & $\rho_{R}$ & $u_{R}$ & $p_{R}$ \\
\hline Contact & 0 & 0.5 & 0 & 1 & 0.25 & 1000 & 0 & $10^{5}$ & 0.01 & 0 & $10^{5}$ \\
Sod & 1 & 0.2 & 0 & 1 & 0.5 & 1 & 0 & 1 & 0.125 & 0 & 0.1 \\
Lax & 2 & 0.14 & 0 & 1 & 0.5 & 0.445 & 1.698 & 3.528 & 0.5 & 0 & 0.571 \\
\hline
\end{tabular}


The reference solution is well captured and the second-order P-IMEX scheme is more accurate, as expected. It is particularly visible for Lax test, where, for the first order scheme, the left rarefaction interacts with the boundary condition because the dissipation is too important (and the number of cells is not sufficiently high). The dissipation is reduced when using a second-order scheme, therefore such spurious interaction does not occur. Moreover the spreading of the shock wave and contact discontinuity is less pronounced with the second order version.
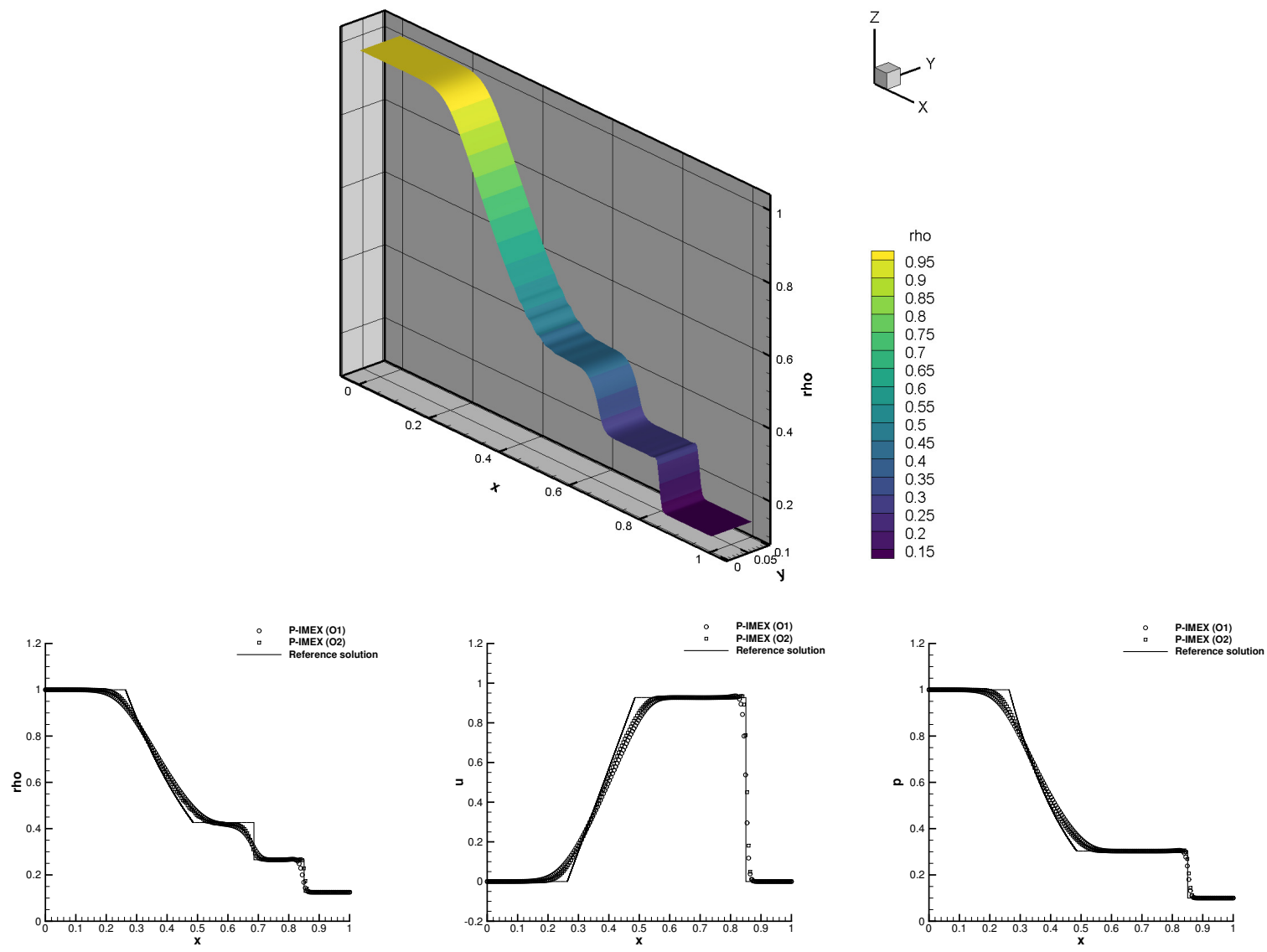

Figure 2: RP1 test case - Sod problem - 1st and 2nd order IMEX schemes - Top: $3 \mathrm{~d}$ view of the density variable - Bottom (left to right): density, velocity and pressure (symbols) vs the exact solution (straight line).

Now, we consider the Riemann problem RP0 described in Table 4 where the initial density is a step-like function of five orders of magnitude, $\rho_{L}-\rho_{R}=10^{5}, p_{L}=p_{R}=10^{5}, u_{L}=u_{R}=u=1$. The Mach number ranges from approximately $2.7 \cdot 10^{-4}$ to $8.5 \cdot 10^{-2}$. In Figure 4 we present the results obtained with the first and second order P-IMEX schemes (density, velocity and pressure with symbols) versus the exact solution (straight line) on the bottom panels. On the top panel we present an extruded three-dimensional view where the color and azimuth scale referred to the density variable. As expected both schemes maintain a constant velocity and pressure, while the initial contact discontinuity is numerically dissipated. The second-order IMEX scheme produces a sharper contact discontinuity.

\subsection{Shock tube problem at different Mach numbers}

We test the behavior of the novel P-IMEX schemes for different values of the Mach number. To this aim, let us consider two regimes, namely $\varepsilon=10^{-2}$ and $\varepsilon=10^{-4}$. The initial condition for the shock tube problem is simply given by

$$
(\rho, u, v, w, p)=\left\{\begin{array}{ll}
(1,0,0,0,1+\varepsilon) & x<x_{s} \\
(1,0,0,0,1) & x \geq x_{s}
\end{array},\right.
$$



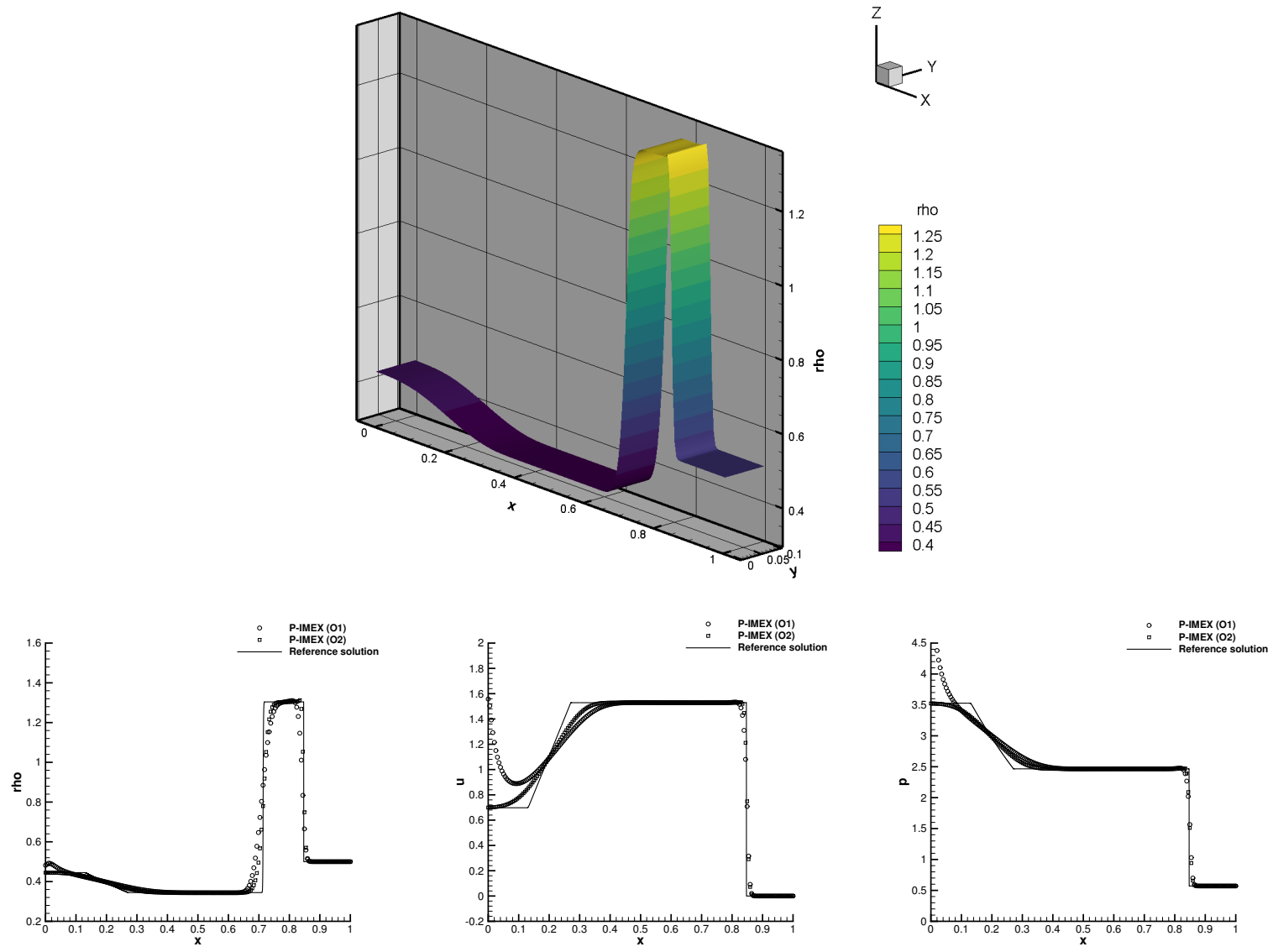

Figure 3: RP2 test case - Lax problem - 1st and 2nd order IMEX schemes — Top: 3d view of the density variable — Bottom (left to right): density, velocity and pressure (symbols) vs the exact solution (straight line). 


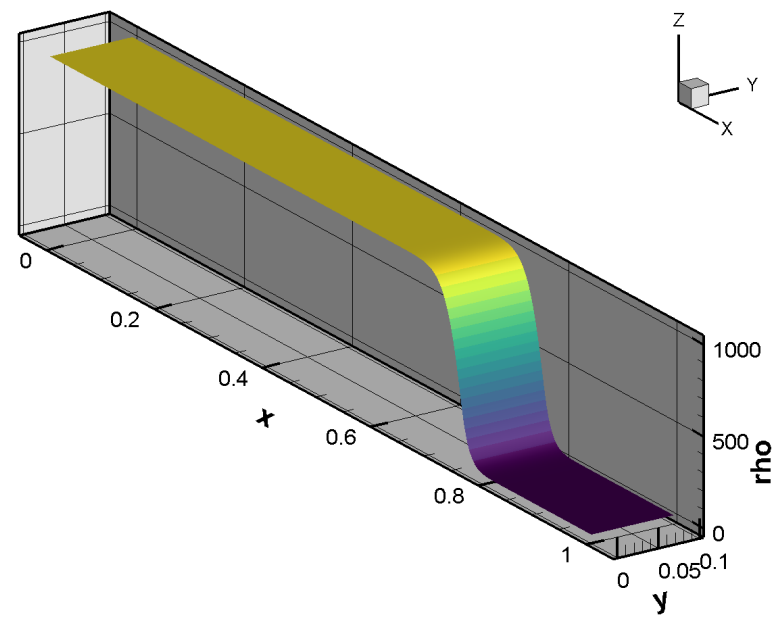

rho: 50100150200250300350400450500550600650700750800850900950
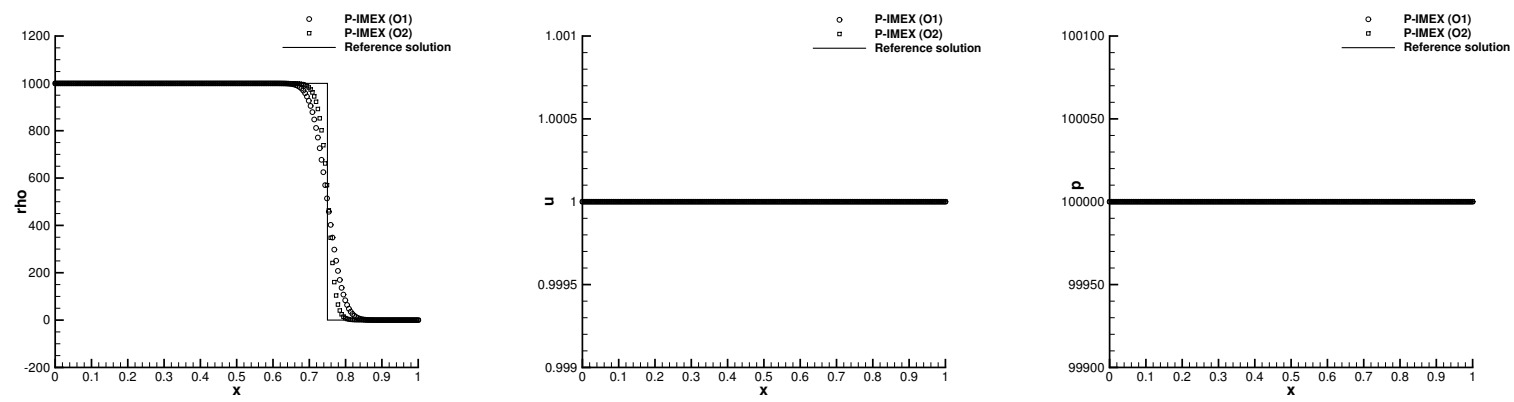

Figure 4: RP0 test case — Moving contact discontinuity — 1st and 2nd order IMEX schemes — Top: 3d view of the density variable — Bottom (left to right): density, velocity and pressure (symbols) vs the exact solution (straight line). 
with $x_{s}=0.5$. The computational domain is again the box $\Omega=[0 ; 1] \times[0 ; 0.1] \times[0 ; 0.1]$, with Dirichlet boundary conditions in $x$-direction and periodic boundaries imposed elsewhere. We always use $N_{y}=N_{z}=5$ control volumes, while a total number of $N_{x}=500$ and $N_{x}=1000$ cells have been employed for $\varepsilon=10^{-2}$ and $\varepsilon=10^{-4}$, respectively. The shock tube problems are run with the following schemes: i) second order explicit (MUSCL-TVD), ii) second order P-IMEX and iii) first order in time and second order in space P-IMEX. A comparison for pressure and momentum distribution is depicted in Figure 5, where one can clearly notice the non-monotonicity of the second order implicit P-IMEX scheme as expected. On the contrary, the first order P-IMEX method provides monotone results but highly diffusive as also expected. The total number of time steps as well as the computational time needed for carrying out the simulations are reported in Table 5. The lower is the Mach number, the more efficient becomes the P-IMEX scheme which only performs one time step to finish the computation. The linear system for the unknown pressure is solved using a matrix-free GMRES algorithm [85] without any preconditioner.

From this test we can conclude that the space limiter is not able to remove overshooting and undershooting of the numerical solution in the case of an implicit time discretization as figured out in [42]. Thus, the violation of the explicit CFL stability condition leads to spurious oscillations which remain bounded but do not disappear. A limiter for this part of the numerical method would be necessary and it will be addressed in the future.

Table 5: Computational time and number of time steps needed for the simulation of the shock tube problems with different schemes

\begin{tabular}{lccc}
\multicolumn{4}{c}{$\varepsilon=10^{-2}$} \\
Scheme & Order in time & CPU time [s] & time steps \\
\hline Explicit & 2 & 1.8125 & 144 \\
Implicit & 2 & 1.8281 & 1 \\
Implicit & 1 & 1.6406 & 1 \\
\hline \multicolumn{5}{c}{$\varepsilon=10^{-4}$} \\
Scheme & Order in time & CPU time [s] & time steps \\
\hline Explicit & 2 & 5.9062 & 261 \\
Implicit & 2 & 3.3437 & 1 \\
Implicit & 1 & 2.9218 & 1 \\
\hline
\end{tabular}

\subsection{Gresho vortex}

In this section we solve the so-called Gresho vortex problem [17] that is a known stationary solution of the Euler equation. The solution, written in polar coordinates, reads

$$
\begin{aligned}
& u_{\theta}(r)= \begin{cases}5 r & 0 \leq r<0.2 \\
2-5 r & 0.2 \leq r<0.4 \\
0 & r \geq 0.4\end{cases} \\
& p(r)= \begin{cases}p_{0}+\frac{25}{2} r^{2} & 0 \leq r<0.2 \\
p_{0}+\frac{25}{2} r^{2}+4[1-5 r-\ln (0.2)+\ln (r)] & 0.2 \leq r<0.4 \\
p_{0}-2+4 \ln (2) & r \geq 0.4\end{cases} \\
& \rho(r)=1
\end{aligned}
$$

where $u_{\theta}(r)$ is the angular velocity, $r=\sqrt{x^{2}+y^{2}}$ is the radius and $p_{0}=\rho / \gamma M^{2}$ is expressed in terms of the Mach number. The velocity field can be obtained from $u_{\theta}$ with a simple rotation, in particular $(u, v)=u_{\theta} / r \cdot(-y, x)$. In order to test the proposed algorithm against different magnitudes of the Mach number, we will use $M=0.1,0.01,0.001$. The computational domain is $\Omega=[-0.5 ; 0.5] \times[-0.5 ; 0.5] \times[-0.05 ; 0.05]$, which is discretized with $N_{x}=N_{y}=80$ and $N_{z}=5$ control volumes. We impose the exact solution (98) as initial and boundary condition. We perform the simulation up to the final time $t_{\text {final }}=0.4 \pi$ using $C F L=0.15$, in order to mimic the same setting adopted in [4]. The comparison between exact and numerical results obtained with the novel second order P-IMEX scheme for several Mach numbers is depicted in Figure 6 for $N_{x}=N_{y}=80$. A very good agreement can be observed at all the values 


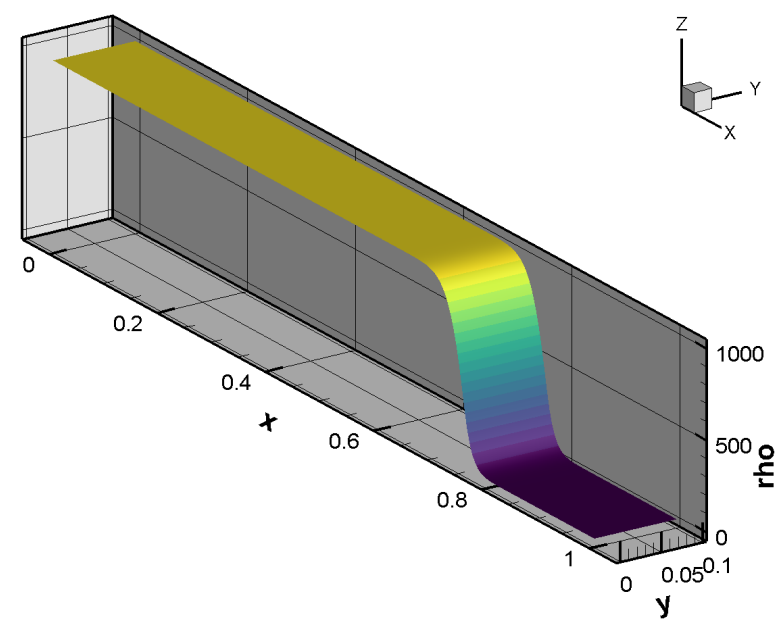

rho: 50100150200250300350400450500550600650700750800850900950
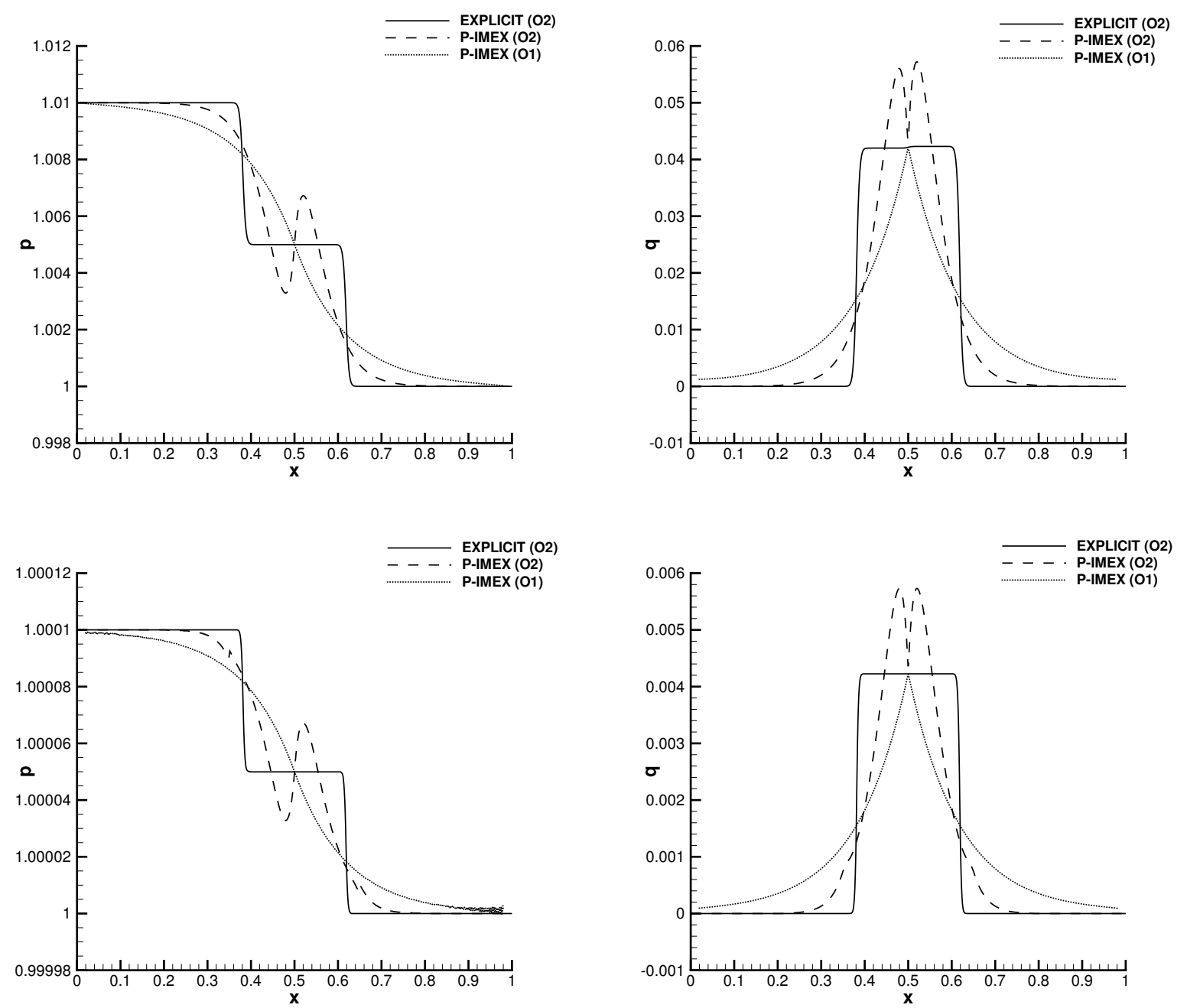

Figure 5: Shock tube problem with $\varepsilon=10^{-2}$ at time $t_{\text {final }}=0.01$ (top row) and with $\varepsilon=10^{-4}$ at time $t_{\text {final }}=0.001$ (bottom row). Pressure (left column) and momentum (right column) distribution are shown for the folfowing schemes: second order explicit (solid line), second order P-IMEX (dashed line) and first order in time P-IMEX (dotted line). 
of the Mach numbers. The resulting dissipation of the total energy $K$ normalized with the initial kinetic energy $K_{0}$ is reported in Figure 7 for all the Mach numbers and for mesh size $h_{1}=1 / 40$ and $h_{2}=1 / 80$. It is clear from Figure 7 that the obtained values of the kinetic energy dissipation do not depend on the chosen Mach number. The resulting profile is also in good agreement with others obtained in literature using an implicit shock capturing scheme, see [4], showing a slightly less dissipative behavior for our P-IMEX schemes.
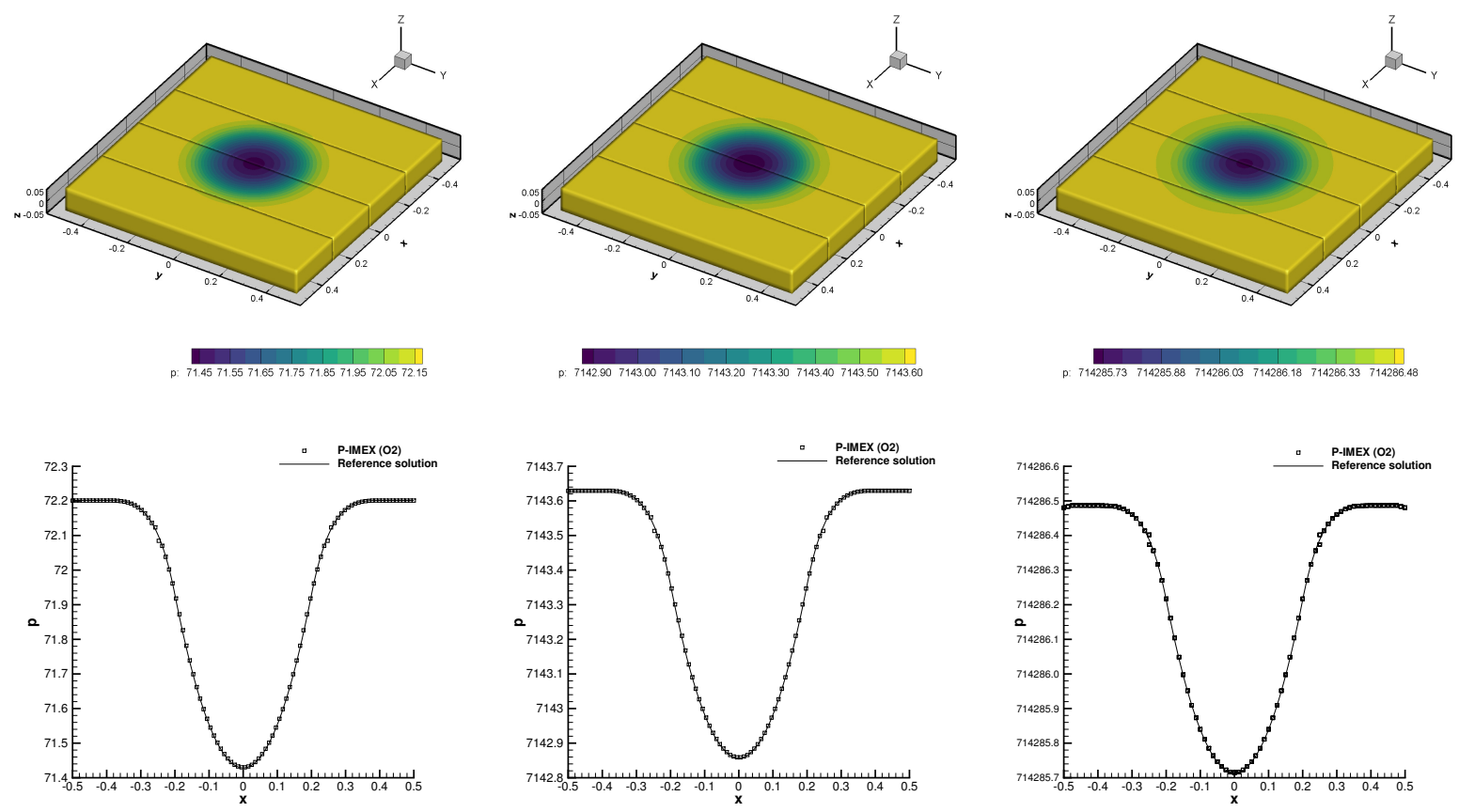

Figure 6: Gresho vortex problem at the final time $t_{\text {final }}=0.4 \pi$ with different Mach number regimes: $M=0.1$ (left), $M=0.01$ (middle), $M=0.001$ (right). Top row: density contours. Bottom row: comparison between numerical and exact pressure distribution.

\subsection{Experimental convergence of the pressure system}

In order to check the numerical convergence of the Picard algorithm needed for the solution of the pressure system (76), we have monitored the residuals at the end of the iterative procedure. Specifically, we have always used a total number of $R_{p}=2$ Picard iterations and the errors $e_{P i c}$ are evaluated in terms of relative pressure that is normalized with the maximum pressure value $p_{\max }$ of the simulation, hence

$$
e_{\text {Pic }}=\frac{\left\|p^{r+1}-p^{r}\right\|_{L_{2}}}{p_{\max }}, \quad r=R_{p} .
$$

The Sod and Lax shock tube problems have been considered as well as the Gresho vortex with three different values of Mach numbers, namely $M=0.1, M=0.01$ and $M=0.001$. The corresponding values of $p_{\max }$ are listed in Table 6 , while Figure 8 depicts the normalized pressure errors. The choice of using a fixed number of Picard iterations yields different error magnitudes, as evident from the average errors reported in Table 6. For flows with shocks and strong discontinuities the errors are bounded in the interval $\left[10^{-2} ; 10^{-4}\right]$, while for smooth flows the pressure errors are always lower than $10^{-4}$ and almost vanish in the low Mach limit (see the Gresho vortex test with $M=0.001$ ).

From this brief experimental analysis, we notice that our simple choice of setting only few Picard iterations leads to relative errors which are smaller than $10^{-2}$ for a reasonably different set of test cases. If the problem involves strong shocks it might be convenient to increase the value of $R_{p}$ and keep monitored the relative pressure errors. In all test 


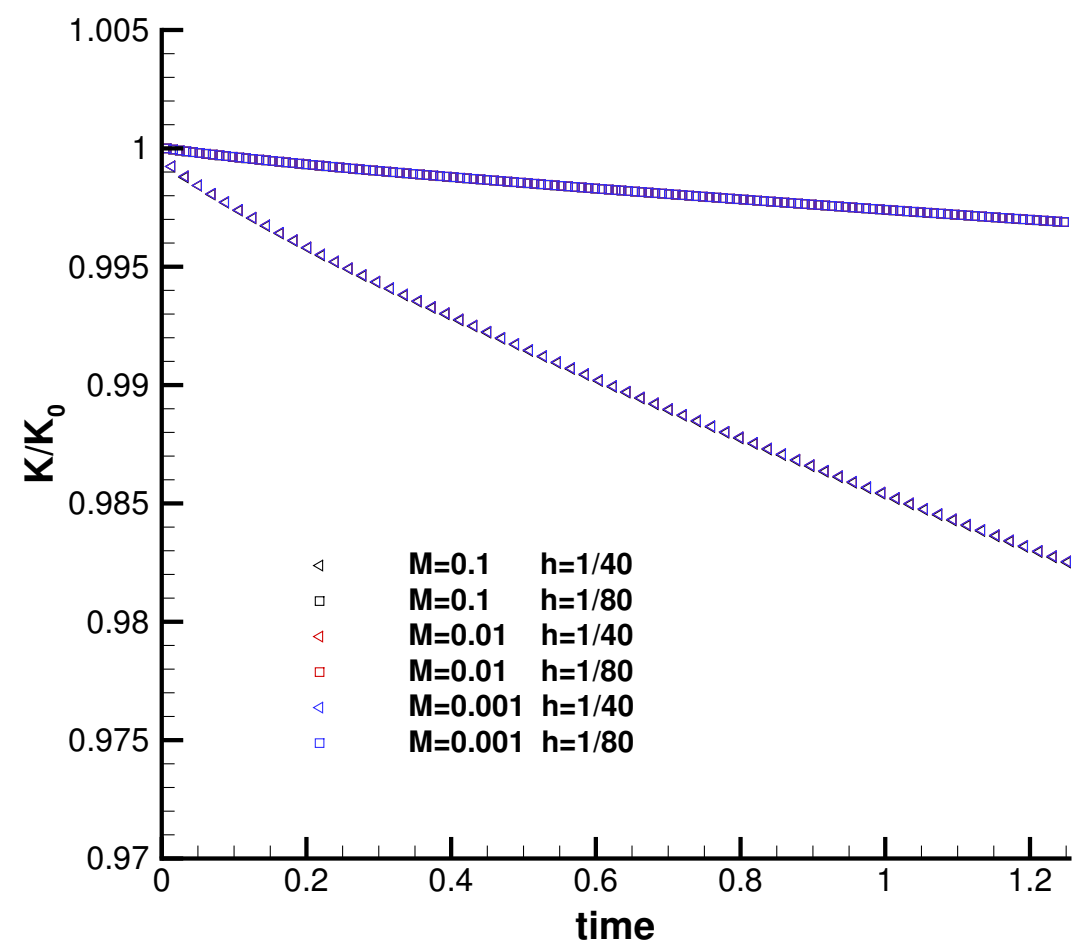

Figure 7: Evolution of the total kinetic energy $K$ normalized with respect to the initial kinetic energy $K_{0}$. Triangle scatters refer to the mesh size $N_{x}=N_{y}=40$, while square scatters refer to the mesh size $N_{x}=N_{y}=80$. Mach number 0.1 (black), 0.01 (red), 0.001 (blue) are considered.

Table 6: Normalizing pressure and error norms of the Picard iterative algorithm for different test cases.

\begin{tabular}{lcccc} 
Name & $p_{\max }$ & time steps & $e_{P i c}$ & $\left\|p^{r+1}-p^{r}\right\|_{L_{2}}$ \\
\hline Lax & $3.5000 \mathrm{E}-00$ & 110 & $7.2089 \mathrm{E}-03$ & $2.5231 \mathrm{E}-02$ \\
Sod & $1.0000 \mathrm{E}-00$ & 97 & $3.5690 \mathrm{E}-03$ & $3.5690 \mathrm{E}-03$ \\
Gresho $(\mathrm{M}=0.1)$ & $7.2200 \mathrm{E}+01$ & 162 & $5.4023 \mathrm{E}-06$ & $3.9005 \mathrm{E}-04$ \\
Gresho $(\mathrm{M}=0.01)$ & $7.1436 \mathrm{E}+03$ & 162 & $5.3240 \mathrm{E}-09$ & $3.8033 \mathrm{E}-05$ \\
Gresho $(\mathrm{M}=0.001)$ & $7.1429 \mathrm{E}+05$ & 162 & $2.5646 \mathrm{E}-12$ & $1.8319 \mathrm{E}-06$ \\
\hline
\end{tabular}



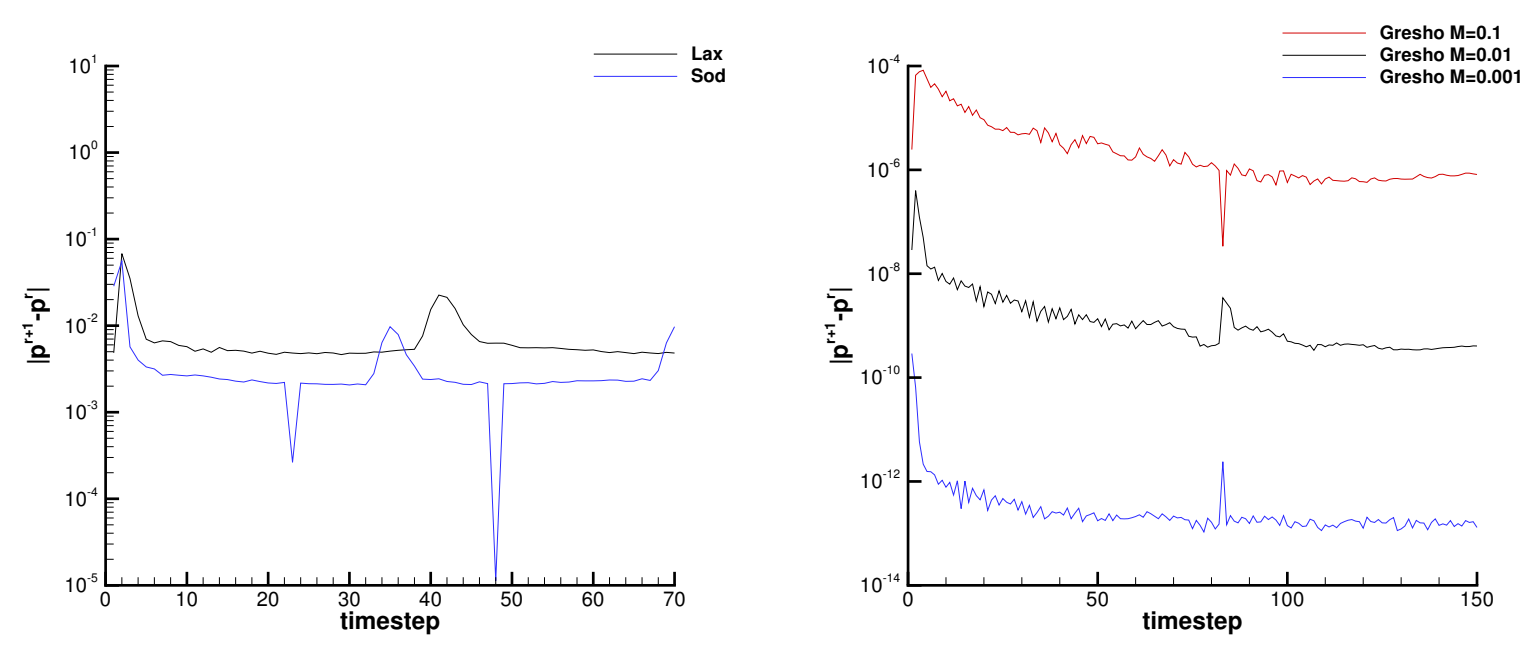

Figure 8: Normalized pressure errors at the end of the Picard iteration for each time step. Left: Lax and Sod shock tube problem. Right: Gresho vortex test case with three different values of the Mach number.

cases shown in this paper we have always set $R_{p}=2$, thus covering a wide set of scenarios affected by different Mach number regimes. As such, this choice seems to be a good compromise between accuracy and efficiency.

\subsection{D Explosion problem}

The proposed second order P-IMEX scheme is checked against the well known three-dimensional explosion problem [94]. This problem can be seen as the three-dimensional extension of the Sod problem already described in section 5.2. The initial condition is given by

$$
(\rho, u, v, w, p)=\left\{\begin{array}{ll}
(1,0,0,0,1) & r<R_{s} \\
(0.125,0,0,0,0.1) & r \geq R_{s}
\end{array},\right.
$$

where $r=\sqrt{x^{2}+y^{2}+z^{2}}$ and $R_{S}=0.5$ is the radius of the sphere. We consider $\Omega=[-1 ; 1]^{3}$ and Dirichlet boundary conditions everywhere. We use $N_{x}=N_{y}=N_{z}=180$ elements so that the total number of elements is $N_{E}=5^{\prime} 832^{\prime} 000$, as depicted in Figure 9. Furthermore $\gamma=1.4$ and $t_{\text {final }}=0.25$. We run the test in parallel with MPI using 64 AMD Opteron(TM) Processor 6272 CPU cores. The reference solution is obtained by considering the angular symmetry of the problem in order to reduce the 3D Euler system to a $1 \mathrm{D}$ system in radial direction with geometric source term, see [94]. The reference solution is actually computed employing a second order TVD scheme with Rusanov-type flux on 20000 radial points. The resulting numerical solution at the final time $t_{\text {final }}=0.25$ is reported in Figure 10 . In the first panel of 10 we plot some isolines for $\rho$ in the semi-space $\{x<0\}$ and show the radial symmetry of the obtained solution. This property is not trivial since the mesh is not radially aligned. Finally, we report the scatter plot of the main variables, considering all the $32^{\prime} 400$ points in the slice $\{(x, y, z) \mid x=0\}$. We observe a very good agreement with the reference solution also for this three-dimensional problem.

\subsection{Forward facing step problem with unsteady Mach number at inflow}

The last test case aims at showing the capability of the P-IMEX scheme to deal with different Mach number regimes within the same simulation. We choose to slightly modify the well-known forward facing step problem [100], which is characterized by reflecting shock waves that travel across the computational domain and interact one with each other. The computational domain is given by $\Omega=\{[0 ; 3] \times[0 ; 0.2] \times[0.2 ; 1]\} \cap\{[0 ; 1] \times[0 ; 0.2] \times[0 ; 0.2]\}$ and is discretized with a total number of cells $N_{x} \times N_{y} \times N_{z}=320 \times 16 \times 16$. The fluid is initialized with

$$
(\rho, u, v, w, p)=\left(1.4,3 \cdot 10^{-4}, 0,0,1\right)
$$




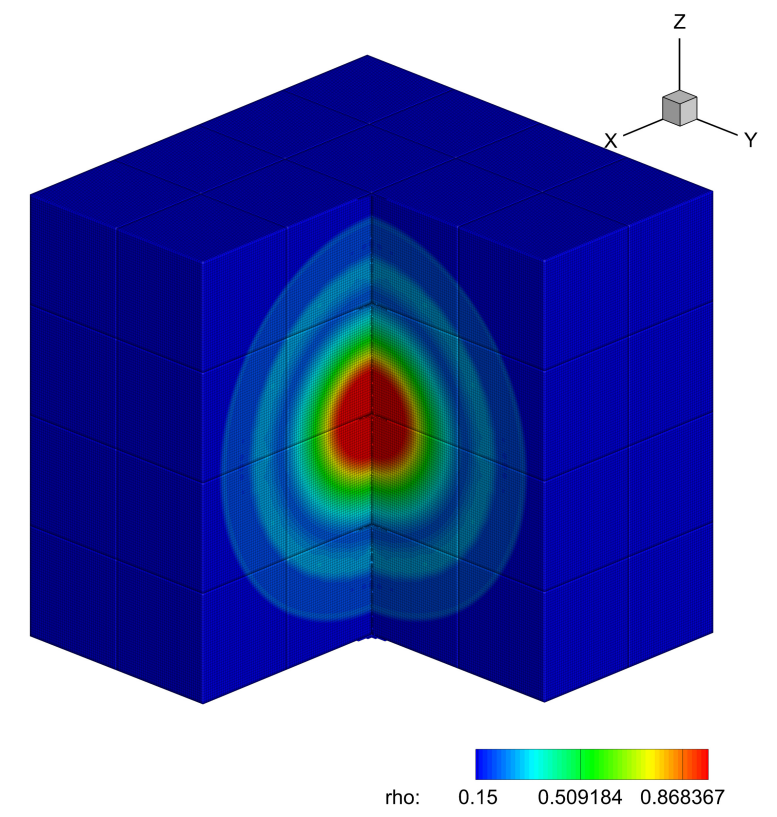

Figure 9: Computational mesh and density contours at time $t=$ for the 3D explosion problem. The computational domain is divided in 64 partitions corresponding to the MPI threads.

so that the flow is in the low Mach regime with initial Mach number $M_{0}=3 \cdot 10^{-4}$. Outflow boundary condition is set at $x=3$, while slip wall boundaries are imposed elsewhere, apart from the inflow at $x=0$. There, we prescribe a time-dependent condition that linearly increases the horizontal velocity $u(t)$ from $3 \cdot 10^{-4}$ to 3 in the time interval $\left[t_{1} ; t_{2}\right]:=[0.75 ; 1.25]$, hence

$$
u(t)=\left\{\begin{array}{lll}
3 \cdot 10^{-4} & \text { if } t \leq t_{1} \\
3 \cdot 10^{-4}+\frac{t-t_{1}}{t_{2}-t_{1}}\left(3-3 \cdot 10^{-4}\right) & \text { if } \quad t_{1}<t \leq t_{1} \\
3 & \text { if } t>t_{2}
\end{array} .\right.
$$

Notice that this setting makes the time step undergo a jump from the low Mach to the high Mach configuration, which is very challenging for the stability and the robustness of the scheme. Transition from high to low Mach is indeed easier regarding the time step limitation. The final time of the simulation is $t_{\text {final }}=4$. Figure 11 reports the Mach number distribution at different output times, namely before, during and after the change of the flow regime due to the unsteady inflow boundary conditions. The corresponding numerical solution for density is then depicted in Figure 12, that highlights the generation of shock waves as the Mach number increases. 

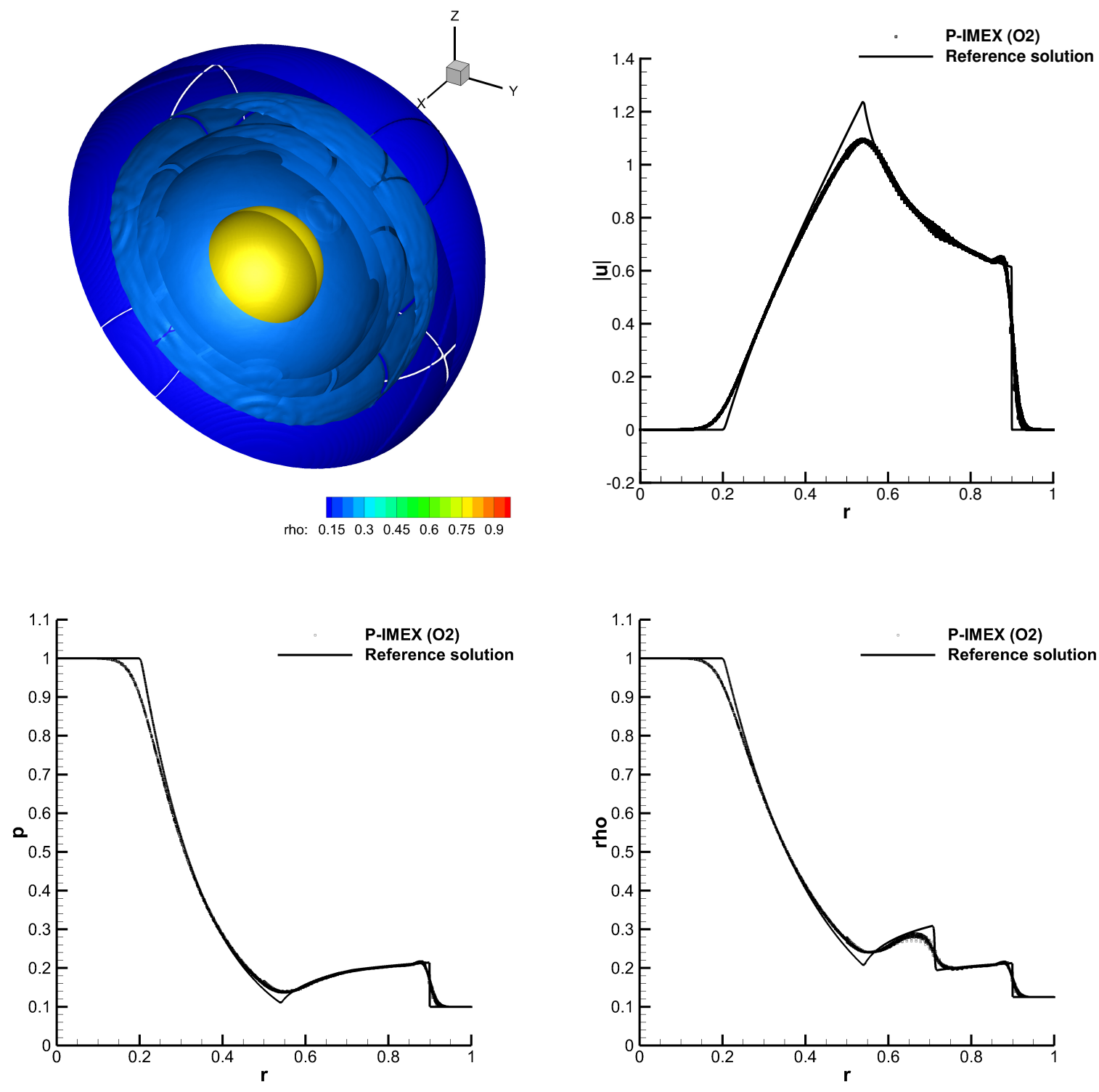

Figure 10: Scatter plot of the numerical solution for the velocity norm $|u|=\sqrt{u^{2}+v^{2}+w^{2}}$, pressure and density obtained with the new P-IMEX method at time $t_{\text {final }}=0.25$ compared with the reference solution. 

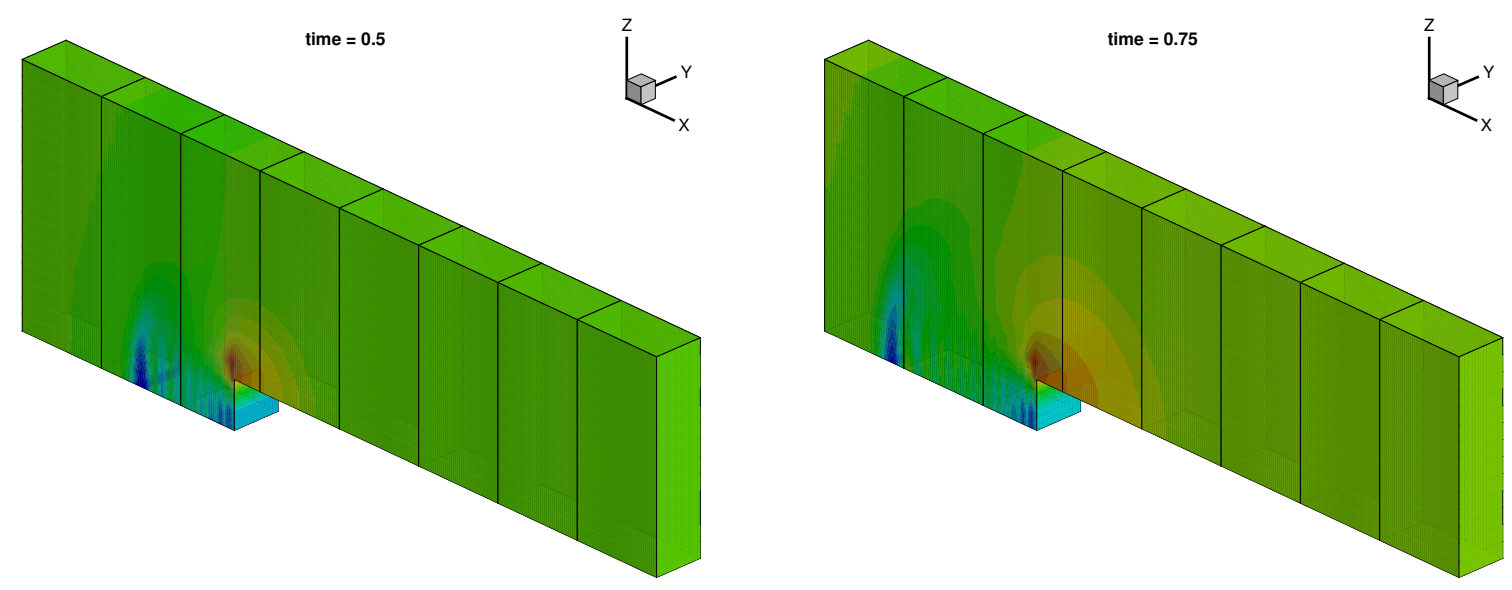

M: $\quad 4 \mathrm{E}-05 \quad 0.00018 \quad 0.00032 \quad 0.00046$

M: $\quad 6 \mathrm{E}-05 \quad 0.00018 \quad 0.0003 \quad 0.00042$
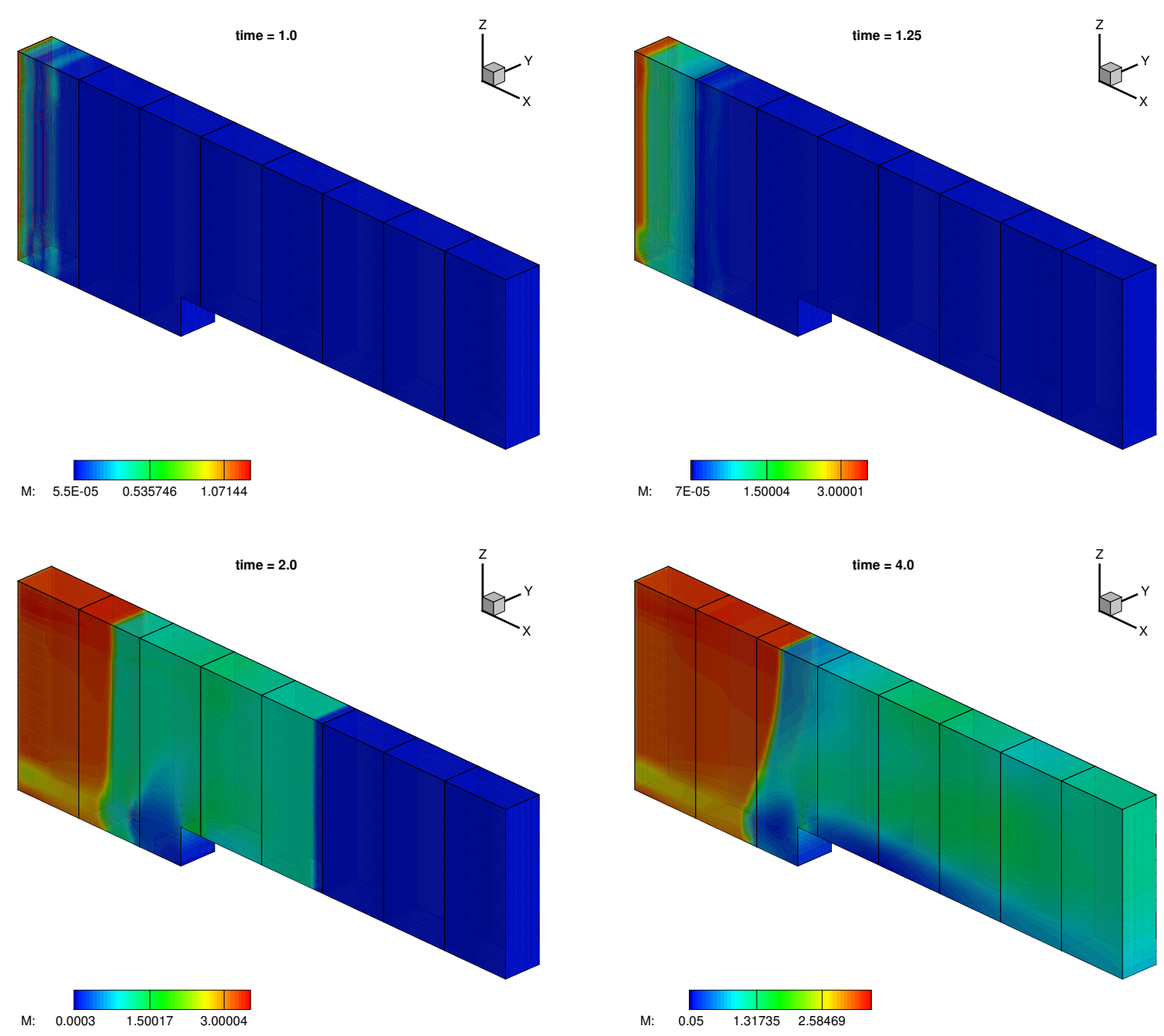

Figure 11: Mach number distribution for the forward facing step problem. Output times (from top left to bottom right): $t=0.5, t=0.75, t=1$, $t=1.25, t=2$ and $t=4$. MPI ranks are separated by black lines. 

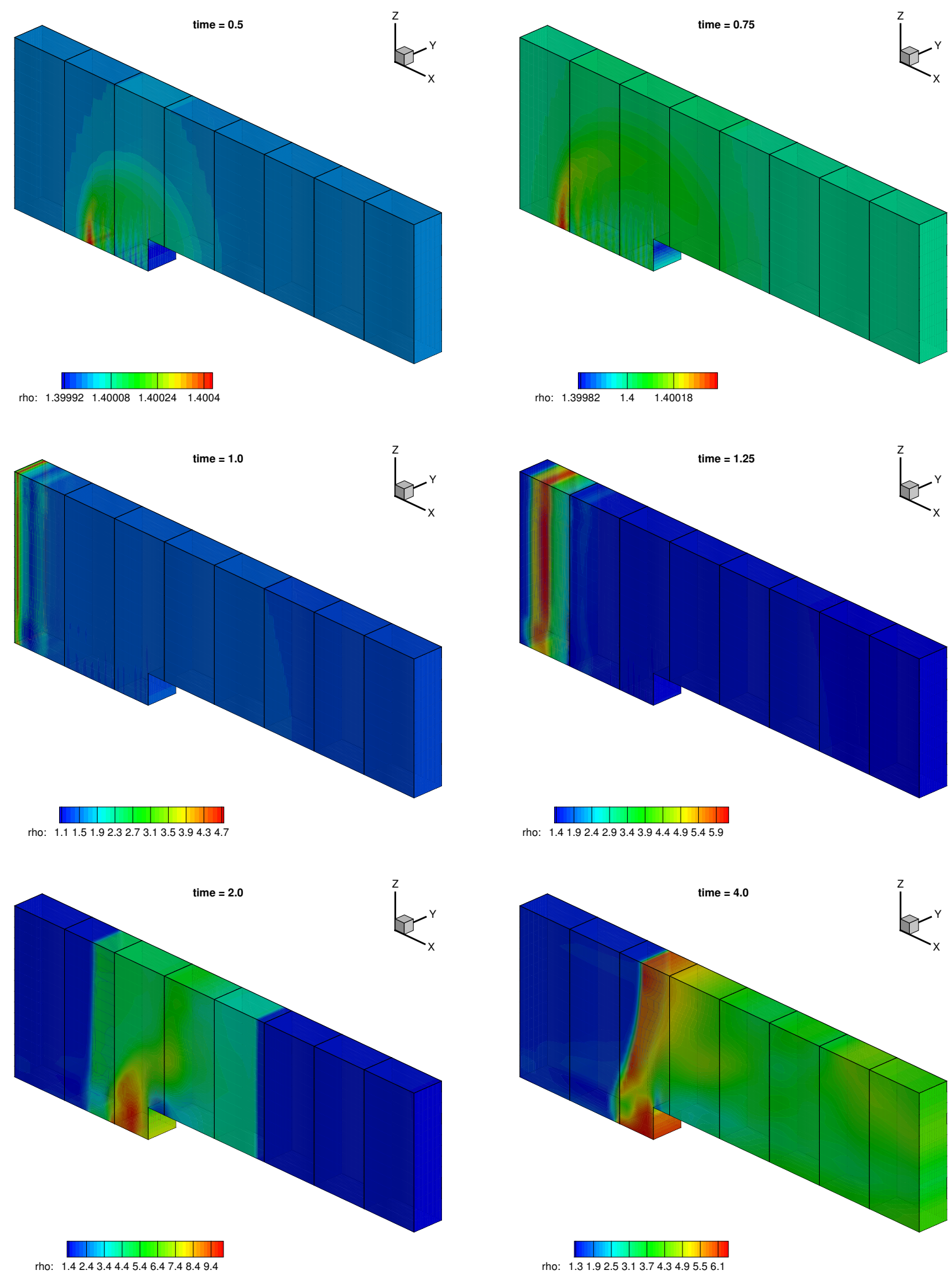

Figure 12: Density distribution for the forward facing step problem. Output times (from top left to bottom right): $t=0.5, t=0.75, t=1, t=1.25$, $t=2$ and $t=4$. MPI ranks are separated by black lines. 


\section{Conclusions and perspectives}

In this article, a second order in space and time finite volume scheme for the solution of the compressible Euler equations in a wide range of Mach numbers has been discussed. The numerical method is based on the splitting of the fluxes into a non stiff and a stiff part. The first one corresponds to the advective contribution while the second one to the pressure part which may be responsible of creating very fast waves compared to the speed of the flow. These two different terms are then discretized, the first one explicitly while the second one implicitly. Second order of accuracy in space has been achieved relying on a piecewise linear reconstruction operator with classical minmod limiter for the explicit fluxes, while central finite difference discretization ensures the same accuracy for the implicit part as well. IMEX Runge-Kutta time stepping guarantees that the novel methods are fully second order accurate even in time.

For the proposed scheme, we showed that the asymptotic preserving property is satisfied and that the theoretical order of accuracy in the semi-discrete case for all values of the Mach number is maintained up to the incompressible limit. An analysis of the properties of the method has also been performed in the fully discretized case for the first order method.

The numerical results demonstrate that a wide spectrum of phenomena can be efficiently solved especially in the low Mach regime by our method, the stability depending only on the speed of the fluid flow and not on the sound speed. The method has been implemented on three-dimensional Cartesian grids and makes use of MPI parallelization. Demonstration of applicability is shown by a set of test problems that span from high to low Mach flows, including convergence studies.

In the future, we plan to extend the presented approach to the compressible Navier-Stokes equations in order to obtain an efficient solver for viscous fluids. Moreover, the development of higher order accurate methods which are consistent with the asymptotic incompressible limit of the Euler equations is the subject of future investigations. Finally, the development of higher order time discretizations which are also able to assure the $L^{\infty}$ stability condition deserves future investigations. We only recall that it has been proved [42] that the $L^{\infty}$ and the Total Variation Diminishing (TVD), also named Strong Stability Preserving (SSP), properties cannot be assured for an unconstrained implicit in time scheme of order greater than one, and, thus, remedy to this negative result should be studied. In [30], a solution constructing high resolution algorithms by combining schemes of different nature has been proposed. We intend to extend and study in details this possibility for the full Euler case in some next future work.

\section{Acknowledgments}

The Authors acknowledge the financial support of French ANR project MOONRISE. The material of this work has been partially obtained during the SHARK-FV conference (Sharing Higher-order Advanced Know-how on Finite Volume www.SHARK-FC.eu/). WB has been partially funded by INdAM (Istituto Nazionale di Alta Matematica Italy) under the program Young researchers funding 2018 and by GNCS (Gruppo Nazionale di Calcolo Scientifico Italy). Finally, the Authors would like to thank the Italian Ministry of Instruction, University and Research (MIUR) to support this research with funds coming from PRIN Project 2017 (No. 2017KKJP4X entitled "Innovative numerical methods for evolutionary partial differential equations and applications").

\section{References}

\section{References}

[1] T. Alazard, Incompressible limit of the nonisentropic Euler equations with the solid wall boundary conditions. Adv. Differential Equations, 10(1), 19-44, 2005.

[2] K. Asano, On the incompressible limit of the compressible Euler equation. Japan J. Appl. Math. 4, 455-488, 1987.

[3] U. M. Ascher, S. J. Ruuth, R. J. Spiteri, Implicit-explicit Runge-Kutta methods for time-dependent partial differential equations. Appl. Numer. Math. 25(2-3) 151-167, 1997, Special issue on time integration (Amsterdam, 1996).

[4] S. Avgerinos, F. Bernard, A. Iollo, G. Russo, Linearly implicit all Mach number shock capturing schemes for the Euler equations, J. Comp. Phys. 393,278-312, 2019.

[5] G. Billet, R. Abgrall An adaptive shock-capturing algorithm for solving unsteady reactive flows, Comput. Fluids 32 (2003) $1473-1495$.

[6] G. Bispen, M. Lukáčová-Medvid'ová, L. Yelash, Asymptotic preserving IMEX finite volume schemes for low Mach number Euler equations with gravitation, J. Comput. Phys. 335, 222-248, 2017. 
[7] M. Boger, F. Jaegle, R. Klein, C.-D. Munz, Coupling of compressible and incompressible flow regions using the multiple pressure variables approach. Math. Methods Appl. Sci. 38 (2015), no. 3, 458-477.

[8] S. Boscarino, G. Russo, On a class of uniformly accurate IMEX Runge-Kutta schemes and applications to hyperbolic systems with relaxation, SIAM J. Sci. Comput. 31(3), 1926-1945, 2009.

[9] S. Boscarino, G. Russo, Flux-explicit IMEX Runge-Kutta schemes for hyperbolic to parabolic relaxation problems, SIAM J. Numer. Anal. 51(1), 163-190, 2013.

[10] S. Boscarino, G. Russo, L. Scandurra, All Mach Number Second Order Semi-Implicit Scheme for the Euler Equations of Gasdynamics, preprint.

[11] W. Boscheri, M. Dumbser, M. Righetti. A Semi-Implicit Scheme for 3D Free Surface Flows with High Order Velocity Reconstruction on Unstructured Voronoi Meshes, Internat. J. Numer. Methods Fluids, 72, 607-631, 2013.

[12] W. Boscheri, G.R. Pisaturo, M. Righetti. High order divergence-free velocity reconstruction for free surface flows on unstructured Voronoi meshes, Internat. J. Numer. Methods Fluids, 90, 296-321, 2019.

[13] C. Bresten, S. Gottlieb, Z. Grant, D. Higgs, D. I. Ketcheson, A. Németh, Explicit strong stability preserving multistep Runge-Kutta methods. Math. Comp. 86, 747-769, 2017.

[14] V. Casulli, P. Zanolli, A nested Newton-type algorithm for finite volume methods solving Richardson equation in mixed form, SIAM J. Sci. Comput. 32 (2009) 2255-2273.

[15] V. Casulli, P. Zanolli, Iterative solutions of mildly nonlinear systems, J. Comput. Appl. Math. 236 (2012) 3937-3947.

[16] C. Chalons, M. Girardin, S. Kokh, Large time step and asymptotic preserving numerical schemes for the gas dynamics equations with source terms, SIAM J. Sci. Comput. 35,2874-2902, 2013.

[17] P. M. Gresho, S.T. Chan On the theory of semi-implicit projection methods for viscous incompressible flow and its implementation via a finite element method that also introduces a nearly consistent mass matrix. Part 2: Implementation, Int. J. Num. Meth. Fluids. 11,621-659, 1990.

[18] C. Chalons, M. Girardin, S. Kokh, An all-regime Lagrange-Projection like scheme for the gas dynamics equations on unstructured meshes, Communications in Computational Physics (CICP), 20(1), 188-233, 2016.

[19] S. Clain, S. Diot, R. Loubère, A high-order finite volume method for systems of conservation laws-Multi-dimensional Optimal Order Detection (MOOD), J. Comput. Phys. 230(10), 4028-4050, 2011.

[20] Ph. Colella, K. Pao, A projection method for low speed flows, J. Comp. Phys. 149, 245-269, 1999.

[21] D. Ghosh and E.M. Constantinescu, Semi-implicit Time Integration of Atmospheric Flows with Characteristic-Based Flux Partitioning, SIAM J. Sci. Comput. 38 (3) A1848-A1875, 2016.

[22] S. Conde, S. Gottlieb, Z. J. Grant, J. N. Shadid, Implicit and Implicit-Explicit Strong Stability Preserving Runge-Kutta Methods with High Linear Order, J. Sci. Comput., 73, 667-690, 2017.

[23] E. M. Constantinescu, A. Sandu, Optimal explicit strong-stability-preserving general linear methods, SIAM J. Sci. Comput. 32, 3130-3150, 2010.

[24] F. Cordier, P. Degond, A. Kumbaro, An Asymptotic-Preserving all-speed scheme for the Euler and Navier Stokes equations, J. Comp. Phys. 231, 5685-5704, 2012

[25] P. Degond, F. Deluzet, A. Sangam, M.-H. Vignal, An Asymptotic Preserving Scheme for the Euler equations in a strong magnetic field, Comp. Phys. 228, 3540-3558, 2009.

[26] P. Degond, M. Tang, All speed scheme for the low Mach number limit of the isentropic Euler equations. Commun. Comput. Phys. 10(1), $1-31,2011$.

[27] P. Degond, S. Jin, J.-G. Liu, Mach-number uniform asymptotic-preserving gauge schemes for compressible flows. Bull. Inst. Math. Acad. Sin. (N.S.) 2 (2007), no. 4, 851-892.

[28] S. Dellacherie, Analysis of Godunov type schemes applied to the compressible Euler system at low Mach number, J. Comp. Phys. 229, 978-1016, 2010.

[29] S. Dellacherie, J. Jung, P. Omnes, Preliminary results for the study of the Godunov Scheme Applied to the Linear Wave Equation with Porosity at Low Mach Number, ESAIM ProcS. 52, 105-126, 2015.

[30] G. Dimarco, R. Loubère, V. Michel-Dansac, M.-H. Vignal, Second order Implicit-Explicit Total Variation Diminishing schemes for the Euler system in the low Mach regime, J. Comput. Phys., 372, 178-201, 2018

[31] G. Dimarco, R. Loubère, M.-H. Vignal, Study of a new asymptotic preserving scheme for the Euler system in the low Mach number limit, SIAM J. Sci. Comput., 39(5), A2099-A2128, 2017.

[32] G. Dimarco, L. Pareschi, Asymptotic-preserving IMEX Runge-Kutta methods for nonlinear kinetic equations, SIAM J. Num. Anal. 1064$1087,2013$.

[33] G. Dimarco, L. Pareschi, High order asymptotic preserving schemes for the Boltzmann equation, Comptes Rendus Mathematique 350, 9, 481-486, 2012.

[34] S. Diot, S. Clain, R. Loubère, Improved detection criteria for the multi-dimensional optimal order detection (MOOD) on unstructured meshes with very high-order polynomials, Comput. \& Fluids, 64, 43-63, 2012.

[35] S. Diot, R. Loubère, and S. Clain, The multidimensional optimal order detection method in the three-dimensional case: very high-order finite volume method for hyperbolic systems, Internat. J. Numer. Methods Fluids, 73(4), 362-392, 2013.

[36] M. Dumbser, V. Casulli, A conservative, weakly nonlinear semi-implicit finite volume scheme for the compressible Navier-Stokes equations with general equation of state, Applied Mathematics and Computation, 2016, vol. 272, issue P2, 479-497

[37] M. Dumbser, D.S. Balsara, M. Tavelli and F. Fambri, A divergence-free semi-implicit finite volume scheme for ideal, viscous, and resistive magnetohydrodynamics, Int. J. Num. Meth. Fluids. 89, 16-42, 2019.

[38] W. E, C.-W. Shu, A Numerical Resolution Study of High Order Essentially Non-oscillatory Schemes Applied to Incompressible Flow, J. Comput. Phys. 110, 39-46, 1994.

[39] R. Eymard, T. Gallouët, R. Herbin, Finite volume methods, Handbook of numerical analysis, Vol. VII, 713-1020, North-Holland, Amsterdam, 
2000.

[40] L. Ferracina, M. N. Spijker, Stepwize restrictions for the total-variation-diminishing property in general Runge-Kutta mathods, SIAM J. Numer. Anal. 42, 1073-1093, 2004.

[41] N. Grenier, J.-P. Vila, P. Villedieu, An accurate low-Mach scheme for a compressible two-fluid model applied to free-surface flows, J. Comp. Phys. 252, 1-19, 2013.

[42] S. Gottlieb, C.-W. Shu, E. Tadmor, Strong stability-preserving high-order time discretization methods, SIAM Rev. 43(1), 89-112, 2001.

[43] H. Guillard, A. Murrone, On the behavior of upwind schemes in the low Mach number limit : II. Godunov type schemes, Comp. \& Fluids, 33, 655-675, 2004.

[44] H. Guillard, C. Viozat, On the behavior of upwind schemes in the low Mach limit, Comp. \& Fluid, 28, 63-86, 1999.

[45] J. Haack, S. Jin, J.G. Liu, An all-speed asymptotic-preserving method for the isentropic Euler and Navier-Stokes equations, Commun. Comput. Phys., 12, 955-980, 2012.

[46] F. H. Harlow, A. Amsden, A numerical fluid dynamics calculation method for all flow speeds, J. Comput. Phys, 8, $197-213,1971$.

[47] D. R. van der Heul, C. Vuik, P. Wesseling, A conservative pressure-correction method for flow at all speeds, Comp. \& Fluids, 32 (2003), pp. 1113-1132.

[48] I. Higueras, N. Happenhofer, O. Koch, F. Kupka, Optimized strong stability preserving IMEX Runge-Kutta methods. J. Comput. Appl. Math. 272 (2014), 116-140.

[49] I. Higueras, Representations of Runge-Kutta methods and strong stability preserving methods, SIAM J. Numer. Anal., 43, 924-948, 2005.

[50] E. Hofer, A partially implicit method for large stiff systems of Ode's with only few equations introducing small time-constants, SIAM J. Numer. Anal. 13, (1976) pp. 645-663.

[51] C. Hu and C.W. Shu. Weighted essentially non-oscillatory schemes on triangular meshes, J. Comput. Phys. 150, 97-127, 1999.

[52] R.I. Issa, A.D.Gosman, A.P. Watkins, The computation of compressible and incompressible flow of fluid with a free surface, Phys. Fluids, 8, 2182-2189, 1965.

[53] A. Jameson, S. Yoon, Multigrid solution of the Euler equations using implicit schemes, AIAA Journal, 24 (1986), $1737-1743$.

[54] A. Jameson, Solution of the Euler equations for two dimensional transonic flow by a multigrid method, Appl. Math. Comp, 13 (1983), $327-355$.

[55] A. Jameson, D.A. Caughey, How Many Steps are Required to Solve the Euler Equations of Steady Compressible Flow: In Search of a Fast Solution Algorithm, AIAA 2001-2673, 15th AIAA Computational Fluid Dynamics Conference, June 11-14, 2001, Anaheim, CA.

[56] S. Jin, Asymptotic preserving (AP) schemes for multiscale kinetic and hyperbolic equations: a review, Riv. Mat. Univ. Parma 3, 177-42,216 2012.

[57] J. Katz, A. Plotkin. Low-Speed Aerodynamics (Cambridge Aerospace Series). Cambridge: Cambridge University Press. (2001).

[58] D. I. Ketcheson, C. B. Macdonald, S. Gottlieb, Optimal implicit strong stability preserving Runge-Kutta methods. Appl. Numer. Math. 59 (2009), no. 2, 373-392.

[59] W. Kheriji, R. Herbin, J.-C. Latché, Pressure correction staggered schemes for barotropic one-phase and two-phase flows, Comp. \& Fluids, $88,524-542,2013$.

[60] S. Klainerman, A. Majda, Singular limits of quasilinear hyperbolic systems with large parameters and the incompressible limit of compressible fluids, Comm. Pure Appl. Math. 34(4), 481-524, 1981

[61] S. Klainerman, A. Majda, Compressible and incompressible fluids, Comm. Pure Appl. Math. 35(5), 629-651, 1982.

[62] R. Klein, Semi-implicit extension of a Godunov-type scheme based on low Mach number asymptotics I: One-dimensional flow, J. Comp. Phys., 121, 213-237, 1995.

[63] B. van Leer, Towards the Ultimate Conservative Difference Scheme, V. A Second Order Sequel to Godunov's Method, J. Com. Phys. 32, 101-136, 1979

[64] R. J. LeVeque, Finite volume methods for hyperbolic problems, Cambridge Texts in Applied Mathematics. Cambridge University Press, Cambridge, 2002.

[65] P.-L. Lions, Mathematical topics in fluid mechanics. Vol. 1, Oxford Lecture Series in Mathematic sand its Applications, vol. 3, The Clarendon Press Oxford University Press, New York, 1996.

[66] P.-L. Lions, N. Masmoudi, Incompressible limit for a viscous compressible fluid, J. Math. Pures Appl. (9), 77(6), 585-627, 1998.

[67] M.-S. Liou, A sequel to AUSM, Part II: AUSM+-up for all speeds, J. Comput. Phys., 214, 137-170, 2006.

[68] G. Métivier, S. Schochet, The incompressible limit of the non-isentropic Euler equations, Arch. Ration. Mech. Anal., 158(1), 61-90, 2001.

[69] G. Métivier, S. Schochet, Averaging theorems for conservative systems and the weakly compressible Euler equations, J. Differential Equations 187 , no. $1,106-183,2003$

[70] C. D. Munz, S. Roller, R. Klein, K. J. Geratz, The extension of incompressible flow solvers to the weakly compressible regime, Comp. Fluid, 32, 173-196, 2002

[71] C. D. Munz, M. Dumbser, S. Roller, Linearized acoustic perturbation equations for low Mach number flow with variable density and temperature, J. Comput. Phys., 224, 352-364, 2007.

[72] S. Noelle, G. Bispen, K.R. Arun, M. Lukáčová-Medvid’ová, C. D. Munz, A weakly asymptotic preserving low Mach number scheme for the Euler equations of gas dynamics. SIAM J. Sci. Comput. 36 (2014), no. 6, B989-B1024.

[73] H. Paillère, C. Viozat, A. Kumbaro, I. Toumi, Comparison of low mach number models for natural convection problems. Heat \& Mass Tran., $36,567-573,2000$.

[74] H. Paillère, C. Core, J. Garcia, On the extension of the AUSM+ scheme to compressible two-fluid models, Computers \& Fluids, 32 891-916, 2003.

[75] L. Pareschi, G. Russo, Implicit-explicit Runge-Kutta schemes for stiff systems of differential equations, In Recent trends in numerical analysis, volume 3 of Adv. Theory Comput. Math. pages 269-288. Nova Sci. Publ., Huntington, NY, 2001.

[76] L. Pareschi, G. Russo, Implicit-Explicit Runge-Kutta schemes and applications to hyperbolic systems with relaxation, J. Sci. Comput. 25(1-2), 129-155, 2005.

[77] J. H. Park, C. D. Munz, Multiple pressure variables methods for fluid flow at all Mach numbers, Int. J. Numer. Meth. Fluid, 49, 905-931, 
2005.

[78] S. V. Patankar, Numerical heat transfer and fluid flow, New York: McGraw-Hill, 1980.

[79] O. Peles, E. Turkel Acceleration Methods for Multi-physics Compressible Flow J. Comput. Phys., 358 (2018) 201-234.

[80] C.-C. Rossow, A Flux-Splitting Scheme for Compressible and Incompressible Flows, J. Comput. Phys., 164, 104-122, 2000

[81] C.-C. Rossow, Convergence acceleration for solving the compressible Navier-Stokes equations, AIAA J., 44, 345-352, 2006.

[82] V.V. Rusanov, Calculation of interaction of non-steady shock waves with obstacles. J. Comput. Math. Phys. USSR 1, 267-279, 1961.

[83] R.C. Swanson, E. Turkel, C.-C. Rossow, Convergence acceleration of Runge-Kutta schemes for solving the Navier-Stokes equations, J. Comput. Phys., 224, 365-388, 2007.

[84] Sani, R. L. and Gresho, P. M. and Lee, R. L. and Griffiths, D. F. and Engelman, M., The cause and cure of the spurious pressures generated by certain FEM solutions of the incompressible Navier-Stokes equations. II, Internat. J. Numer. Methods Fluids, 171-204, 1981.

[85] Y. Saad, M. Schultz, GMRES: a generalized minimal residual algorithm for solving nonsymmetric linear systems, SIAM J. Sci. Stat. Comput. 7, 856-869, 1986.

[86] S. Schochet, The compressible Euler equations in a bounded domain: existence of solutions and the incompressible limit, Comm. Math. Phys., 104(1), 49-75, 1986.

[87] A. H. Shapiro, The Dynamics and Thermodynamics of Compressible Fluid Flow, May 1953.

[88] J. A. Smoller, J. L. Johnson. Global solutions for an extended class of hyperbolic systems of conservation laws, Arch. Rational Mech. Anal. 32, 169-189, 1969.

[89] H. Song, Energy SSP-IMEX Runge-Kutta methods for the Cahn-Hilliard equation. J. Comput. Appl. Math. 292 (2016), 576-590

[90] M. Tang, Second order all speed method for the isentropic Euler equations. Kinet. Relat. Models 5 (2012), no. 1, 155-184.

[91] M. Tavelli and M. Dumbser, A staggered space-time discontinuous Galerkin method for the incompressible Navier-Stokes equations on two-dimensional triangular meshes, Comput. \& Fluids, 119, 235-249, 2015.

[92] M. Tavelli and M. Dumbser, A pressure-based semi-implicit space-time discontinuous Galerkin method on staggered unstructured meshes for the solution of the compressible Navier-Stokes equations at all Mach numbers, J. Comp. Phys., 341, 341-376, 2017.

[93] M. Tavelli and M. Dumbser, A staggered, space-time discontinuous Galerkin method for the three-dimensional incompressible Navier-Stokes equations on unstructured tetrahedral meshes, J. Comp. Phys., 319, 294-323, 2016.

[94] E. F. Toro, Riemann solvers and numerical methods for fluid dynamics. A practical introduction, Springer-Verlag, Berlin, third edition, 2009.

[95] E.F. Toro, M.E. Vázquez-Cendón, Flux splitting schemes for the Euler equations, Comput. Fluids 70 (2012) 1-12.

[96] E. Turkel, Preconditioned methods for solving the incompressible and low speed compressible equations, J. Comp. Phys., 72, 277-298, 1987.

[97] E. Turkel and V.N. Vatsa, Local Preconditioners for Steady State and Dual Time-Stepping, ESAIM: M2AN, 39, 515-536, 2005.

[98] B. van Leer, Towards the Ultimate Conservative Difference Scheme V: A second Order sequel to Godunov's Method, J. Comp. Phys., 32, 101-136, 1979.

[99] F. Vilar, P.-H. Maire, R. Abgrall, Cell-centered discontinuous Galerkin discretizations for two-dimensional scalar conservation laws on unstructured grids and for one-dimensional Lagrangian hydrodynamics, Comput. \& Fluids, 46, 498-504, 2011.

[100] P. Woodward and P. Colella, The numerical simulation of two-dimensional fluid flow with strong shocks, J. Comp. Phys., 54, 115-173, 1984.

[101] J. Zeifang, J. Schütz, K. Kaiser, A. Beck, M. Lukáčová-Medvid’ová and S. Noelle, A Novel Full-Euler Low Mach Number IMEX Splitting, Commun. Comput. Phys. (CICP), 27, 292-320, 2020. 